\title{
Optimal reinsurance and investment strategies for insurers with mispricing and model ambiguity *
}

\author{
Ailing $\mathrm{Gu}^{\mathrm{a}}$, Frederi G. Viens ${ }^{\mathrm{b}}$, Bo Yi ${ }^{\mathrm{c}, \mathrm{d}, *}$ \\ ${ }^{a}$ School of Applied Mathematics, Guangdong University of Technology, Guangzhou 510520, P.R. China \\ ${ }^{b}$ Department of Statistics and Probability, Michigan State University, East Lansing, MI 48824, USA \\ ${ }^{c}$ Research Center, Shenzhen Venture Capital Group CO.LTD, Shenzhen 518048, PR China \\ ${ }^{d}$ School of Economics and Management, Tsinghua University, Beijing 100084, PR China
}

\begin{abstract}
We discuss optimal proportional reinsurance-investment problems for an insurer with mispricing and model ambiguity under a complex stochastic environment. The surplus process is described by a classical Cramér-Lundberg (C-L) model and the financial market contains a pair of mispriced stocks, a risk-free asset, and a market index. The insurer is ambiguityaverse and has specific modeling risk aversion preferences for the financial market's diffusion term and insurance model's jump term respectively. The statistical arbitrage opportunities which are afforded by our mispricing model feature are particularly timely in the insurance investment context for markets in Hong Kong and mainland China. Similar to Maenhout (2004), we incorporate the ambiguity aversion and formulate an optimal robust reinsuranceinvestment problem. By employing the dynamic programming approach, we derive the explicit optimal robust reinsurance-investment strategy and optimal value function. We provide numerical illustrations to analyze the behavior of our formulas and make practical recommendations. By studying our portfolio allocation sensitivity to various parameters, among other things, we uncover and analyze complex behavior resulting from asymmetry between the mean-reversion rates of the mispriced stocks. We also define and analyze various utility losses which explain the importance of ambiguity aversion, surplus-jump, mispricing, and reinsurance in our model. Some of our findings include: the importance of taking advantage of mispricing for medium and long-term investment strategies, whereas decisions with a one-year horizon or less can usually ignore mispricing arbitrage opportunities; a recommendation never to ignore jumps in the surplus model; and an analysis of how crucial it is to incorporate ambiguity, especially for moderate time horizons.

Keywords: Proportional reinsurance; Robust control; Optimal investment strategy; Utility function; Mispricing.
\end{abstract}

${ }^{\star}$ This research is supported by grants from the National Natural Science Foundation of China (Nos. 71501050, 71301031), National Science Foundation of Guangdong Province of China (No. 2014A030310195).

${ }^{*}$ Corresponding author.

Email addresses: ailing727@sohu.com (Ailing Gu), byi@szvc.com.cn (Bo Yi)

Preprint submitted to Elsevier

(C) 2016. This manuscript version is made available under the Elsevier user license

http://www.elsevier.com/open-access/userlicense/1.0/ 


\section{Introduction}

Reinsurance and investment are the main measures insurers use to control their risk and gain profits from surplus. The optimal reinsurance and investment problem has attracted considerable attention from the fields of actuarial and financial mathematics. Typically, one of three types of insurance models are used: the Cramér-Lundberg (C-L) model (see Schmidli H. (2001), Zhou (2005)), a diffusion model (see Grandell (1991), Chen et al. (2010), Shen and Zeng (2014) ) and a jump-diffusion model (see Gerber (1970), Gu et al. (2014)). In this paper we adopt the classical C-L model to describe the surplus for the insurers.

Although many scholars have investigated optimal reinsurance and investment strategies for insurers, it is a notorious fact in the practice of portfolio management that crucial parameters under jump-diffusion are difficult to estimate with any satisfactory precision. As a consequence, the Ambiguity-Averse Insurer (AAI) will look for a methodology to handle this modeling uncertainty. Rather than make ad-hoc decisions about how much error is contained in the surplus and financial market models, the AAI may instead consider some alternative models which are close to the estimated model. This more systematic method has been successfully implemented over the last 15 years in quantitative investment finance, for portfolio selection and asset pricing with model uncertainty or model misspecification, and has seen some recent applications in insurance. Some of these prominent results applied to finance include the following. Anderson et al. (1999) introduced ambiguity-aversion into the Lucas model, and formulated alternative models. Uppal and Wang (2003) extended Anderson et al. (1999), and developed a framework which allows investors to consider the level of ambiguity. Maenhout (2004, 2006) optimized an inter-temporal consumption problem with ambiguity, and derived closed-form expressions for the optimal strategies under a "homothetic robustness" assumption about ambiguity aversion. That work finds the optimal portfolio choice under model uncertainty and stochastic premiums, and provides a methodology to measure the quantitative effect of model uncertainty. Liu et al. (2005) discussed the role of ambiguity aversion in option pricing. Subsequently, based on the work of Maenhout (2004, 2006), Liu (2010) studied the robust consumption and portfolio choice problem; Munk and Rubtsov (2014) investigated portfolio management with stochastic interest rates

and inflation ambiguity; Yi et al. (2015) studied optimal portfolio selection problem with ambiguity aversion in a financial market with mispricing.

All the above-mentioned papers are limited to studying ambiguity in portfolio allocation, which applies strictly to finance. However, in the context of insurance and reinsurance, the same modeling uncertainty, i.e. the same ambiguity, exists in the expected surplus of insurers. Therefore, an ambiguity-averse insurer should consider both sources of ambiguity in making optimal decisions. A limited number of works have emerged recently on this topic. 
Zhang and Siu (2009) and Korn et al. (2012) used stochastic differential games to study the optimal reinsurance-investment problem with ambiguity; Yi et al. (2013, 2015) studied the optimal reinsurance-investment strategy with ambiguity aversion under expected utility maximization and mean-variance criterion.

However, this literature focuses on the ambiguity of diffusion risk; the ambiguity caused by the intensity of jumps has been ignored. Recently, Branger and Larsen (2013) and Jin and Zeng (2013) investigated the optimization problem for an ambiguity-averse investor under a market with event-risk, the results show that the ambiguity aversion to jump risk has a strong impact on the optimal investment strategy. Zeng et al. (2016) also found that the jump risk has the important impact on the optimal reinsurance strategy. This motivates us to ask about ambiguity in the intensity parameter of the jump-risk in the classical C-L model, which is a new element we have addressed in this paper. In the C-L model, in which insurance claims are responsible for jumps, an AAI will wish to incorporate uncertainty in jump modeling. In this paper, since we incorporates jump risk, our model also considers this aversion to the uncertainty in modeling the jump risk (ambiguity aversion in jump risk). We choose to incorporate this ambiguity aversion in a way which is consistent with the robust control suggested by Maenhout (2004) which was originally designed for diffusive ambiguity. We consider alternative models which are close to the reference model, in the sense that their drifts differ from the reference model, so that their distance to it can be quantified in a straightforward way and used to implement a penalty based on this distance. This framework allows us to derive an optimal robust reinsurance-investment strategy. Our results show that the proportional reinsurance will change with the jump-risk ambiguity aversion level and the AAI will want to purchase more reinsurance if she shows more aversion to jump risk. By comparing the utility functions in the jump model and in a diffusion approximation model, the AAI will typically prefer to use a jump model and seek a more conservative strategy rather than to pursue a strategy which would otherwise yield higher utility if there were no jump-risk ambiguity.

We also introduce a further model feature which is not present in the existing literature on reinsurance-investment problems: we investigate possible investments in a financial market with a so-called mispricing phenomenon. This is the first paper in which an insurance investor may choose to optimize investments by exploiting mispricing. It is of timely significance to Chinese insurance inverstors, since in 2015, the Chinese government opened up simultaneous investment in mainland China and Hong Kong financial market, which means that a mainland China investor is allowed to invest in designated Hong Kong stocks and vice versa. Based on the reality that a number of stocks of national corporations are traded in both Shanghai and Hong Kong markets, and the price gap between the stocks for the same product in two markets is already significant, this policy may lead to sustained discrepancies for these stock prices in the two financial markets. Indeed the new policy, which was 
arguably introduced in part for the sake of increasing mutual liquidity, will be accompanied with persistent frictions which will continue to affect most investors on these markets.

Mispricing is a price difference between a pair of assets, where two assets with identical or nearly identical contingent claim values ought to have the same or close to the same price during a same trading period, but do not because of the aforementioned persistence of frictions. For example, as we were just describing in general terms, there exists mispricing in some Chinese companies (such as Bank of China, Agriculture Bank of China, and others) traded on both Chinese stock exchanges as share A and Hong Kong stock exchanges as shares H. A common strategy for taking advantage of mispricing is a "long-short" (L-S) strategy, which takes positions of equal size but opposite signs either in portfolio weight or in number of shares, see Liu and Longstaff (2004) and Jurek and Yang (2006). This kind of strategy is used as long-term arbitrage opportunities, and does not consider temporary diversification benefits. In order to exploit mispricing optimally, Liu and timmermann (2013) established a portfolio maximization framework and derived optimal investment strategies.

Yi et al. (2015) were first to consider a portfolio problem with model ambiguity and mispricing. In this paper, we seek optimal reinsurance-investment strategies for an insurer in an ambiguity-averse insurance model and an ambiguity-averse financial market, the latter of which allows insurers' investments in pairs of mispriced assets for the first time. Given that there are a number of stocks from exchanges in Shanghai, Hong Kong, and Shenzhen which can now be traded simultaneously in China, insurers in China now have these new opportunities to allocate their surpluses. In particular, $\mathrm{A}+\mathrm{H}$ shares with the discount/premium mispricing advantage modeled herein will appeals to insurers seeking to capitalize on such statistical arbitrages which are currently stable. Thus, our paper's consideration of mispricing in the Chinese context of insurance surplus investment is timely and important.

Our insurance surplus model is described by the C-L model, our financial market consist of one risk-free asset, a market index and a pair of stocks with mispricing. To summarize our novel contributions compared to existing literature: (i) Ours is the first paper in which an AAI may consider a robust reinsurance-investment problem in a financial market with mispricing, which we illustrate by using parameters calibrated to the current popular mispriced markets in China and Hong Kong, which is also a first for applications to Chinese insurance companies. As such our paper's results may provide important technological guidance for Chinese insurance management decisions. With the performance gauged by exponential utility functions, we find that the absolute positions in stocks will decrease with respect to aversion to financial market modeling ambiguity. The retention level of the AAI will decrease when she becomes more ambiguity-averse to jump-risk modeling. Moreover, the AAI tends to buy more reinsurance in short investment horizons, and less so with longer-term horizons. (ii) Our paper studies the impact of ambiguity aversion, mispricing, reinsurance and jump modeling in the surplus, on utility losses. In the view of profit, to avoid large losses, the AAI 
cannot ignore mispricing and reinsurance. Particularly, the utility loss would increase with the investment horizon. In terms of safety, model ambiguity and surplus-jump modeling are beneficial for the insurer, since they will make her take more conservative strategies to avoid the sudden occurrence of risk exposure. This kind of risk depends on the ambiguity aversion levels and the intensity of the claims (see Figure 3 and 4).

The rest of this paper is organized as follows. The financial market and the insurance model are described in Section 2. In Section 3 the optimal robust reinsurance-investment problem is established. Optimal reinsurance-investment strategies and their corresponding optimal value functions are given in Section 4. Section 5 provides numerical example to illustrate our results and analyze the utility loss due to ignoring ambiguity aversion, mispricing, reinsurance, and thhe jump term in the surplus. Section 6 concludes this paper.

\section{Economy and assumptions}

In this section, we formulate a continuous-time financial model where the insurers can trade in the financial market and in the insurance market with no taxes or fees. Let $\left(\Omega, \mathcal{F},\left\{\mathcal{F}_{t}, 0 \leq t \leq T\right\}, P\right)$ be a complete probability space, in which $T>0$ is a fixed constant, representing the time horizon, and $\left\{\mathcal{F}_{t}, 0 \leq t \leq T\right\}$ is a filtration satisfying the usual condition (it contains all $P$-null sets and is right continuous). All stochastic processes given in the following are assumed to be adapted to this filtration.

\subsection{Surplus process}

Assume that an insurer's surplus process is described by the classical Cramér-Lundberg (C-L) model: without reinsurance and investment, the insurer's surplus $R$ is given by (see Bäuerle (2005))

$$
d R(t)=p d t-d \sum_{i=1}^{N_{t}} Y_{i}
$$

where $p$ is the premium rate; $\left\{N_{t}, 0 \leq t \leq T\right\}$ is a homogeneous Poisson process with intensity $\lambda>0$ (thus $N_{t}$ represents the number of claims which have arrived up to time $t$ ), $Y_{i}$ represents the size of the $i$ th claim and the claim sizes $Y_{1}, Y_{2}, \ldots$ are assumed to be independent and identically distributed (i.i.d.) positive random variables with common distribution

$F$ having finite first-order moment $\mu_{\infty}$ and second-order moment $\sigma_{\infty}^{2}$. Consequently $\sum_{i=1}^{N_{t}} Y_{i}$ is a compound Poisson process representing the cumulative amount of claims in time interval $[0, t]$. Assume that the premium rate $p$ is calculated according to the expected value principle, i.e., $p=(1+\eta) \lambda \mu_{\infty}$, where $\eta>0$ is the insurer's relative safety loading.

In this paper, the insurer can purchase proportional reinsurance or acquire new business, to decrease the business risk or increase her profits. For each $t \in[0, T]$, the retention level of proportional reinsurance is associated with a risk exposure $q(t) \in[0,+\infty)$. When 
$q(t) \in(0,1]$, it means the insurer purchases proportional reinsurance. In this case, for each claim, the insurer only pays $100 q \%$ while the remaining $100(1-q) \%$ is picked up by the reinsurer. In return, for each claim, the insurer should divert part of the premium to the reinsurer. We assume that the insurer pay the reinsurance premium continuously to the reinsurer. When $q(t) \in(1, \infty)$, it means the insurer acquires new business from the other insurers as a reinsurer. Here, we assume that reinsurance is not inexpensive, i.e., the safety loading of the reinsurer $\theta$ is greater than the safety loading of the insurer $\eta$. Assuming we use the expected value principle again for the reinsurer, the reinsurance premium rate calculates as $(1-q(t))(1+\theta) \lambda \mu_{\infty}$. The reinsurance strategy is denoted by $\{q(t): t \in[0, T]\}$. Putting it all together, the insurer's surplus $R$ with proportional reinsurance is governed by

$$
\begin{aligned}
d R(t) & =[\eta-\theta+(1+\theta) q(t)] \lambda \mu_{\infty} d t-q(t) d \sum_{i=1}^{N_{t}} Y_{i} \\
& =[\eta-\theta+(1+\theta) q(t)] \lambda \mu_{\infty} d t-q(t) \int_{R^{+}} y(t) N(d t, d y)
\end{aligned}
$$

where $N(\cdot, \cdot)$ defined on $\Omega \times[0, T] \times R^{+}$is a Poisson random measure used to represent the compound Poisson process with elements $\left\{N_{t}, Y_{i}\right\}$. If we denote $\nu(d t, d y):=\lambda d t d F(y)$, then the expectation of this process is represented as

$$
E\left[\sum_{i=1}^{N_{t}} Y_{i}\right]=\int_{0}^{t} \int_{R^{+}} y \nu(d t, d y)
$$

Moreover $\nu$ is the compensator of random measure $N(\cdot, \cdot)$. Therefore, the compensated mea-

sure $\widetilde{N}$ of the compound Poisson process $\sum_{i=1}^{N_{t}} Y_{i}$ can be given by $\widetilde{N}(\cdot, \cdot):=N(\cdot, \cdot)-\nu(\cdot, \cdot)$. Moreover, we can rewrite the surplus of the insurer as:

$$
d R(t)=[\eta-\theta+(1+\theta) q(t)] \lambda \mu_{\infty} d t-q(t) \int_{R^{+}} y(t) \widetilde{N}(d t, d y)-q(t) \int_{R^{+}} y(t) \lambda d t d F(y)
$$

\subsection{Financial market}

We assume that financial market consists of one risk-free asset, the market index, and a pair of stocks with mispricing (two price processes for the same asset on two distinct stock markets). The price process of the risk-free asset solves $d S_{0}(t)=r S_{0}(t) d t$ where we assume the risk-free interest rate $r>0$ is constant. The price process of the market index follows a geometric Brownia motion

$$
\frac{d P_{m}(t)}{P_{m}(t)}=\left(r+\mu_{m}\right) d t+\sigma_{m} d B_{t}
$$


where the market risk premium $\mu_{m}$ and the market volatility $\sigma_{m}$ are positive constants, and $\left\{B_{t}\right\}$ is a standard Brownian motion. The two mispriced price processes are modeled as a pair of stocks $P_{1}$ and $P_{2}$ which are coupled via the pricing error

$$
X(t):=\ln \frac{P_{1}(t)}{P_{2}(t)}:
$$

we assume the vector $\left(P_{1}, P_{2}\right)$ solves the system of stochastic differential equations

$$
\begin{aligned}
& \frac{d P_{1}(t)}{P_{1}(t)}=\left(r+\mu_{m} \beta\right) d t+\beta \sigma_{m} d B_{t}+\sigma d Z_{t}+b d Z_{1 t}-l_{1} X(t) d t, P_{1}(0)=P_{10} \\
& \frac{d P_{2}(t)}{P_{2}(t)}=\left(r+\mu_{m} \beta\right) d t+\beta \sigma_{m} d B_{t}+\sigma d Z_{t}+b d Z_{2 t}+l_{2} X(t) d t, P_{2}(0)=P_{20}
\end{aligned}
$$

in which $l_{1}, l_{2}, \sigma, b, \beta$ are constant parameters. The term $\beta \sigma_{m} d B_{t}$ represents the systematic risk of the market, while $\sigma d Z_{t}+b d Z_{i t}$ describe the idiosyncratic risk of stock $i, \sigma d Z_{t}$ describes the common risk, while the individual risk $b d Z_{i t}$ is generated by the asset $i, i=1,2$. The term $l_{i} X(t) d t$ shows the effect of mispricing on $i$ th stock's price via the pricing error $X(t)$ defined above. Based on the Equation (3), ordinary stochastic calculus implies that the dynamics of the pricing erro $X(t)$ is given by the following equation

$$
d X(t)=-\left(l_{1}+l_{2}\right) X(t) d t+b d Z_{1 t}-b d Z_{2 t}, X(0)=x_{0}
$$

Equation (4) identifies $X$ as a Gaussian Mean Reverting process (also known as an OrnsteinUhlenbeck process), implying that the investment opportunities are time-varying. The coefficient $l_{1}$ and $l_{2}$ in Equation (4) can be interpreted as liquidity terms since they control the rate of mean reversion, in such a way that lower liquidity slows down the reversion of the pricing error towards its long-term mean of zero. These coefficients ought not equal zero simultaneously; otherwise, the pair of stocks would represents two assets with the same expected rate of return but higher risk than the market index, which is unrealistic. We assume that $l_{1}+l_{2}>0$, the same assumption can be found in Liu and Timmermann (2013). This assumption ensures that the market is stable, since the corresponding $X$ in (4) is ergodic and asymptotically stationary (under the contrary assumption, $X$ would explode exponentially). Moreover, all standard Brownian motions $\{B(t)\},\left\{Z_{t}\right\},\left\{Z_{1 t}\right\}$ and $\left\{Z_{2 t}\right\}$ are independent each other and all are independent of $N(d y, d t)$.

\subsection{Wealth process}

In addition to reinsurance, the insurer may invest her surplus in the financial market: she can purchase shares of risk-free assets, market index risky assets, and the pair of mispriced stocks. We denote her reinsurance-investment strategy as $\pi:=\{\pi(t), 0 \leq t \leq T\}=$ $\left\{\left(q(t), \pi_{m}(t), \pi_{1}(t), \pi_{2}(t)\right), t \in[0, T]\right\}$, where $\{q(t)\}_{t \in[0, T]}$ represents the reinsurance strategy , $u(t):=\left(\pi_{m}(t), \pi_{1}(t), \pi_{2}(t)\right)$ represents the investment strategy, and $\pi_{m}(t), \pi_{1}(t)$, and $\pi_{2}(t)$ 
denote the investment proportion in the market index and the two stocks, respectively, hence the remainder, $1-\pi_{m}(t)-\pi_{1}(t)-\pi_{2}(t)$, is invested in (or borrowed from) the risk-free asset. As a result of adopting the strategy $\pi$, the corresponding reserve (wealth) of the insurer is described by

$$
\begin{aligned}
d W^{\pi}(t) & =W^{\pi}(t)\left[r+\mu_{m} \bar{u}(t)+X(t)\left(\pi_{2}(t) l_{2}-\pi_{1}(t) l_{1}\right)\right] d t+W^{\pi}(t)\left[\sigma_{m} \bar{u}(t) d B_{t}\right. \\
& \left.+\sigma\left(\pi_{1}(t)+\pi_{2}(t)\right) d Z_{t}+b\left(\pi_{1}(t) d Z_{1 t}+\pi_{2}(t) d Z_{2 t}\right)\right] \\
& +(\eta-\theta+(1+\theta) q(t)) \lambda \mu_{\infty} d t-q(t) \int_{R^{+}} y(t) N(d y, d t), W(0)=w_{0}
\end{aligned}
$$

where we use the shorthand notation $\bar{u}(t):=\pi_{m}(t)+\beta\left(\pi_{1}(t)+\pi_{2}(t)\right)$ and $w_{0}$ is the initial wealth of the insurer.

\section{Robust problem with mispricing}

\subsection{Utility function}

In most of the existing literature, the insurer is assumed to be an ambiguity-neutral investor (ANI), who believes completely in a model determined by statistical estimation or a calibration procedure; we call this kind of this model the reference model, and use the letter $P$ to denote its probability measure. If the insurer is assumed to have CARA utility, in traditional reinsurance and investment models, the ANI maximizes the expected terminal wealth as

$$
\max _{\pi \in \Pi_{0}} E_{0}^{P}\left[U\left(W^{\pi}(T)\right)\right]=\max _{\pi \in \Pi_{0}} E_{0}^{P}\left[-\frac{1}{\gamma} \exp \left\{-\gamma W^{\pi}(T)\right]\right.
$$

where $\gamma>0$ represents the absolute risk aversion coefficient, $\Pi_{0}$ is the set of admissible strategies for an ambiguity-neutral insurer $(\mathrm{ANI})$ in a given market and $E_{t}^{P}[\cdot]=E^{P}\left[\cdot \mid \mathcal{F}_{t}\right]$ represents the conditional expectation under the probability measure $P$. In order to incorporate the ambiguity, we assume that the insurer does not have full confidence in the reference model $P$, because of uncertainty on the parameters (misspecification error); she recognizes that $P$ is only an approximation of reality, and thus takes into consideration some alternatives to $P$. Every alternative model is characterized by a stochastic process $\epsilon$ ( $\epsilon$ will be defined in the later) and the associated probability measure $Q$, which is equivalent to the reference measure $P$. For notational convenience, we denote this class of probability measures by $\mathcal{Q}$ :

$$
\mathcal{Q}:=\{Q: Q \sim P\}
$$

Definition 3.1. A strategy $\pi=\left\{q(t), \pi_{m}(t), \pi_{1}(t), \pi_{2}(t)\right\}_{t \in[0, T]}$ is said to be admissible, if

(1) $\forall t \in[0, T], q(t) \in[0, \infty)$;

(2) $\pi$ is predictable with respect to $\left\{\mathcal{F}_{t}\right\}$ and $E^{Q^{*}}\left[\int_{0}^{T}\|v(t)\|^{2} d t\right]<\infty$, where $\|v(t)\|^{2}=$ $q(t)^{2}+\pi_{m}^{2}(t)+\pi_{1}(t)^{2}+\pi_{2}^{2}(t) ;$

(3) $\forall(t, w) \in[0, T] \times R$, Equation (5) has an unique solution $\left\{W^{\pi}(t)\right\}_{t \in[0, T]}$ with $E_{t, w}^{Q^{*}}\left[U\left(W^{\pi}(t)\right)\right]<$ 
$\infty$, where $Q^{*}$ is our models' worst-case-scenario probability measure which will be described later under appropriate penalization.

Denote by $\Pi$ the set of all admissible strategies. Similarly, we can define the set of admissible strategy $\Pi_{0}, \Pi^{1}$, $\hat{\Pi}$ and $\tilde{\Pi}$ with no ambiguity aversion, no surplus-jump, no mispricing and no reinsurance, respectively.

The change of measure from $P$ to $Q$ can be defined by its Radon-Nikodym derivative, i.e., there exists a progressively measurable process $\epsilon=(\varphi(t), \phi(t))$, such that every $Q$ in $\mathcal{Q}$ satisfies

$$
\frac{d Q}{d P}=\xi(T)
$$

and $\xi(t)$ has the following form

$$
\frac{d \xi(t)}{\xi(t)}=-\varphi(t) d \mathcal{Z}_{t}+\int_{R}(\phi(t)-1) \tilde{N}(d t, d y)
$$

where $\varphi=\left(h_{m}(t), h(t), h_{1}(t), h_{2}(t)\right)$, and $\|\varphi(t)\|^{2}=h_{m}^{2}(t)+h_{1}^{2}(t)+h_{2}^{2}(t)+h^{2}(t)$. We assume that the measure $Q$ is different from the original measure $P$ only in the intensity of claims arrival and in the drifts in the financial models; both measures $P$ and $Q$ share the same claim size ditribution $F$ and the same financial volatilities. Here $\lambda \phi$ is the claim intensity of the Poisson process under the new measure $Q$; and $d \mathcal{Z}_{t}=\left(d B_{t}, d Z_{t}, d Z_{1 t}, d Z_{2 t}\right)$. According to Girsanov's Theorem, the following processes with $Q$-superscripts are Brownian motions under $Q$ :

$$
\begin{aligned}
& d B_{t}^{Q}:=d B_{t}+h_{m}(t) d t, d Z_{t}^{Q}:=d Z_{t}+h(t) d t \\
& d Z_{1 t}^{Q}:=d Z_{1 t}+h_{1}(t) d t, d Z_{2 t}^{Q}:=d Z_{2 t}+h_{2}(t) d t
\end{aligned}
$$

Moreover, under $Q$,

$$
\widetilde{N}(d t, d y)=N(d y, d t)-\lambda^{Q} d F(y) d t=N(d y, d t)-\lambda \phi d F(y) d t
$$

is a martingale. Furthermore, the surplus process of the insurer under the measure $Q$ with reinsurance-investment strategy satisfies the following dynamics :

$$
\begin{aligned}
d W^{\pi}(t) & =W^{\pi}(t)\left[r+\mu_{m} \bar{u}(t)+X(t)\left(\pi_{2}(t) l_{2}-\pi_{1}(t) l_{1}\right)-h_{m}(t) \sigma_{m} \bar{u}(t)\right. \\
& \left.-\sigma h(t)\left(\pi_{1}(t)+\pi_{2}(t)\right)-b\left(\pi_{1}(t) h_{1}(t)+\pi_{2}(t) h_{2}(t)\right)\right] d t \\
& +W^{\pi}(t)\left[\sigma_{m} \bar{u}(t) d B_{t}^{Q}+\sigma\left(\pi_{1}(t)+\pi_{2}(t)\right) d Z_{t}^{Q}+b\left(\pi_{1}(t) d Z_{1 t}^{Q}+\pi_{2}(t) d Z_{2 t}^{Q}\right)\right] \\
& +(\eta-\theta+(1+\theta) q(t)) \lambda \mu_{\infty} d t-q(t) \int_{R^{+}} y(\tilde{N}(d t, d y)+\phi \lambda d F(y) d t)
\end{aligned}
$$

As mentioned above, the reference model $P$ is an approximation of the true model. Even though the insurer is suspicions towards $P$, she is reluctant to deviate too far from $P$. Thus, in the process of considering alternative models $Q$, we use the distance between such $Q_{\mathrm{s}}$ 
and the reference $P$ by penalizing any deviations. Therefore, any choice of an alternative measure $Q$ will consider a trade-off between not being completely dependent on the reference $P$ and not deviating from $P$ too much. Based on the surplus process $(8)$, we formulate a robust control problem, inspired by Maenhout (2004), as follows:

$$
J(t, w, x)=\sup _{\pi \in \Pi} \inf _{Q \in \mathcal{Q}} E_{t, w, x}^{Q}\left\{-\frac{\delta}{\gamma} e^{-\gamma w(T)}+\int_{t}^{T}\left(\frac{R_{1}(s)}{\psi_{1}(s)}+\frac{R_{2}(s)}{\psi_{2}(s)}\right) d s\right\}
$$

where the second term of (9) represents for the deviation from the reference measure, and $R_{1}(t)$ and $R_{2}(t)$ measure the relative entropy arising from diffusion modeling risk and jump modeling risk. According to Hansen and Sargent (2001) and Zeng et al. (2016), the relative entropies for each of these two types of modeling deviations are given by

$$
R_{1}(s)=\frac{1}{2}\|\varphi(s)\|^{2}, R_{2}(s)=(\phi(s) \ln \phi(s)-\phi(s)+1) \lambda .
$$

Here $\psi_{1}(t)$ and $\psi_{2}(t)$ stand for preference parameters for ambiguity aversion to the financial market models and to the insurer's claim intensity. Another interpretation of $\psi_{1}(t)$ and $\psi_{2}(t)$ are as measures of the true model information which the insurer has; the greater $\psi_{1}(t)$ and $\psi_{2}(t)$ are, the less the insurer knows about the true models, the less faith in the reference model she has, and the more the worst case model $Q^{*}$ will deviate from the reference model (this $Q^{*}$, which is defined for a fixed strategy $\pi$ as a measure which minimizes the infemum in (9), will be computed in the next section). For convenience, similar to Maenhout (2004), we assume that $\psi_{1}(s)$ and $\psi_{2}(s)$ are non-negative and state-dependent functions which are inversely proportional to the value function :

$$
\psi_{1}(s)=-\frac{\alpha_{1}}{\gamma J(t, w, x)}, \psi_{2}(s)=-\frac{\alpha_{2}}{\gamma J(t, w, x)},
$$

where $\alpha_{1} \geq 0, \alpha_{2} \geq 0$ represent the insurer's ambiguity aversion levels to the diffusion modeling risk and jump modeling risk.

Remark 3.1. (i) In the case of $\alpha_{1}(t)=\alpha_{2}(t) \equiv 0$, the model (9) degenerates to the ANI model, where the insurer believes that the reference model is the true model. That is to say,

$$
\inf _{Q \in \mathcal{Q}} E_{t, w, x}^{Q}\left\{-\frac{\delta}{\gamma} e^{-\gamma w(T)}+\int_{t}^{T}\left(\frac{R_{1}(s)}{\psi_{1}(s)}+\frac{R_{2}(s)}{\psi_{2}(s)}\right) d s\right\}=E_{t, w, x}^{P}\left\{-\frac{\delta}{\gamma} e^{-\gamma w(T)}\right\} .
$$

Thus, $\int_{t}^{T}\left(\frac{R_{1}(s)}{\psi_{1}(s)}+\frac{R_{2}(s)}{\psi_{2}(s)}\right) d s \equiv 0$. In order to ensure this point, since all terms in the integrand are positive, $R_{1}(t)=R_{2}(t) \equiv 0$ must be satisfied for all $t$.

(ii) In the case of $\alpha_{1}(t)=\alpha_{2}(t)=\infty$, the term $\frac{R_{1}(t)}{\psi_{1}(t)}+\frac{R_{2}(t)}{\psi_{2}(t)}=0$ no matter what the entropies $R_{1}$ and $R_{2}$ are. The insurer has no information about true model and lose all 
confidence in the reference model. Model (9) degenerates to an ill-posed robust problem.

(iii) By allowing the penalty term in (9) to depend separately on the two relative entropies, we thus allow the insurer to specify her ambiguity aversion levels separately for the finance and insurance models.

\section{Solution to the models}

In this section, we solve the optimization problem (9) and derive the optimal value function $J$ and optimal strategy $\pi^{*}$. Moreover, by choosing special values for our model parameters, we describe some special case of (9).

\subsection{Optimal robust reinsurance-investment strategy}

In this section, the insurer aims to obtain robustness by seeking optimal robust strategy under the worst-case scenario. Specially, we fix an admissible strategy $\pi$ to get worse case measure $Q^{*}(\pi) \in \mathcal{Q}$, then optimal robust strategy is attained by

$$
\sup _{\pi \in \Pi} E_{t, w, x}^{Q^{*}(\pi)}\left\{-\frac{\delta}{\gamma} e^{-\gamma w(T)}+\int_{t}^{T}\left(\frac{R_{1}(s)}{\psi_{1}(s)}+\frac{R_{2}(s)}{\psi_{2}(s)}\right) d s\right\}
$$

where we have chosen to work with wealth and pricing error $(w, x)$ as our state variables. The reader can check that using $\left(w, p_{1}, p_{2}\right)$ as the state variables leads to the same solution; hence the use of $x$ results in a reduction of the state space.

In the following, we use dynamic programming to find the worst-case measure $Q^{*}$ and the optimal robust strategy in (9). Note that minimizing over $Q \in \mathcal{Q}$ is equivalent to minimizing over all values of the time- and state-dependent $\epsilon=(\varphi, \phi)$. Based on the robust HamiltonJacobi-Bellman equation (see Anderson et al. (2003), Maenhout (2006)) and the properties of jump terms in the HJB framework (see Bäuerle (2005)), we have

$$
\begin{aligned}
\operatorname{supinf}_{\pi \in \Pi}\left\{\begin{array}{l}
J_{t}\{ \\
\end{array}\right. & J_{w}(\eta-\theta+(1+\theta) q(t)) \lambda \mu_{\infty}+J_{w} w\left[r+\left(\mu_{m}-\sigma_{m} h_{m}(t)\right) \bar{u}(t)\right. \\
& -\sigma h(t)\left(\pi_{1}(t)+\pi_{2}(t)\right)-b\left(\pi_{1}(t) h_{1}(t)+\pi_{2}(t) h_{2}(t)\right)+x\left(\pi_{2}(t) l_{2}\right. \\
& \left.\left.-\pi_{1}(t) l_{1}\right)\right]+\frac{1}{2} J_{w w} w^{2}\left(\sigma_{m}^{2} \bar{u}^{2}(t)+\sigma^{2}\left(\pi_{1}(t)+\pi_{2}(t)\right)^{2}+b^{2}\left(\pi_{1}^{2}(t)+\pi_{2}^{2}(t)\right)\right) \\
& +J_{w x} w b^{2}\left(\pi_{1}(t)-\pi_{2}(t)\right)-J_{x}\left[\left(l_{1}+l_{2}\right) x+b h_{1}(t)-b h_{2}(t)\right]+J_{x x} b^{2} \\
& +\phi(t) \lambda E^{Q}[J(t, w-q(t) Y, x)-J(t, w, x)]-\frac{\gamma J}{\alpha_{1}} \frac{1}{2}\|\varphi(t)\|^{2} \\
& \left.-\frac{\gamma J}{\alpha_{2}}(\phi(t) \ln \phi(t)-\phi(t)+1) \lambda\right\}=0
\end{aligned}
$$

with the boundary condition $J(T, w, x)=-\frac{1}{\gamma} \exp \{-\gamma w\}$, and $J_{t}, J_{w}, J_{w w}, J_{x}, J_{x x}, J_{w x}$ represent the value function's partial derivative w.r.t. the corresponding variables. 
In order to obtain the solution $J$ of $(11)$, we conjecture that $J(t, w, x)$ has the following ansatz form

$$
J(t, w, x)=-\frac{\delta}{\gamma} \exp \left\{-\gamma\left[P(t) w+Q(t)+\frac{1}{2} M(t) x^{2}\right]\right\}
$$

with the boundary condition $P(T)=1, Q(T)=M(T)=0$. A direct calculation yields partial derivatives

$$
\begin{aligned}
& J_{t}=-\gamma\left(P^{\prime}(t) w+Q^{\prime}(t)+\frac{1}{2} M^{\prime}(t) x^{2}\right) J, J_{w}=-\gamma P(t) J, J_{w w}=\gamma^{2} P(t)^{2} J, \\
& J_{x}=-\gamma M(t) x J, J_{x x}=\gamma M(t) J\left(\gamma M(t) x^{2}-1\right), J_{w x}=\gamma^{2} P(t) M(t) x J, \\
& \frac{J_{w}}{J}=-\gamma P(t), \frac{J_{x}}{J}=-\gamma M(t) x, \frac{J}{J_{w}}=-\frac{1}{\gamma P(t)}, \frac{J_{w w}}{J_{w}}=-\gamma P(t), \\
& J(t, w-q Y, x)-J(t, w, x)=J(t, w, x)(\exp \{\gamma P(t) q Y\}-1),
\end{aligned}
$$

where $J=J(t, w, x)$. According to the first-order conditions for $h_{m}, h, h_{1}, h_{2}$, we have

$$
\begin{aligned}
& -J_{w} \sigma_{m} w \bar{u}(t)-\frac{1}{\alpha_{1}} \gamma J h_{m}(t)=0 ; \\
& -J_{w} w \sigma\left(\pi_{1}(t)+\pi_{2}(t)\right)-\frac{1}{\alpha_{1}} \gamma J h(t)=0 ; \\
& -J_{w} b \pi_{1}(t)-J_{x} b-\frac{1}{\alpha_{1}} \gamma J h_{1}(t)=0 ; \\
& -J_{w} w b \pi_{2}(t)+J_{x} b-\frac{1}{\alpha_{1}} \gamma J h_{2}(t)=0
\end{aligned}
$$

i.e.,

$$
\begin{gathered}
h_{m}^{*}(t)=-\frac{\alpha_{1}}{\gamma J} w \sigma_{m} \bar{u} J_{w} ; h^{*}(t)=-\frac{\alpha_{1}}{\gamma J} w \sigma\left(\pi_{1}(t)+\pi_{2}(t)\right) J_{w} ; \\
h_{1}^{*}(t)=-\frac{\alpha_{1}}{\gamma J} b\left(w \pi_{1}(t) J_{w}+J_{x}\right) ; h_{2}^{*}(t)=-\frac{\alpha_{1}}{\gamma J} b\left(w \pi_{2}(t) J_{w}-J_{x}\right)
\end{gathered}
$$

Similarly, by the first condition for $\phi$

$$
\lambda E^{P}[J(t, w-q(t) Y, x)-J(t, w, x)]-\frac{1}{\alpha_{2}} \gamma J(t, w, x) \lambda \ln \phi=0
$$

i.e.,

$$
\phi^{*}=\exp \left\{\frac{\alpha_{2}}{\gamma J(t, w, x)} E^{p}[J(t, w-q Y, x)-J(t, w, x)]\right\}=\exp \left\{\frac{\alpha_{2}}{\gamma} E^{P}\left[e^{P(t) q(t) \gamma Y}-1\right]\right\} .
$$

Therefore, we have determined the worst case measure $Q^{*}$ under the ansatz (12), my specifying that $Q^{*}$ is defined via $(6)$ and $\epsilon=\epsilon^{*}:=\left(\varphi^{*}, \phi^{*}\right)$ with $\varphi^{*}=\left(h_{m}^{*}, h^{*}, h_{1}^{*}, h_{2}^{*}\right)$.

Next, we seek the optimal robust reinsurance-investment strategy. Substituting (14), 
(15), (16) into the HJB equation (11) obtains

$$
\begin{aligned}
& \sup _{\pi \in \Pi}\left\{J_{t}+J_{w}\left[(\eta-\theta+(1+\theta) q(t)) \lambda \mu_{\infty}\right]+w r+\mu_{m} w \bar{u}(t)+w x\left(\pi_{2}(t) l_{2}-\pi_{1}(t) l_{1}\right)\right] \\
& +\frac{1}{2} J_{w w} w^{2}\left(\sigma_{m}^{2} \bar{u}^{2}(t)+\sigma^{2}\left(\pi_{1}(t)+\pi_{2}(t)\right)^{2}+b^{2}\left(\pi_{1}^{2}(t)+\pi_{2}^{2}(t)\right)\right)+J_{w x} w b^{2}\left(\pi_{1}(t)-\pi_{2}(t)\right) \\
& -J_{x}\left(l_{1}+l_{2}\right) x+J_{x x} b^{2}+\frac{\alpha_{1}}{2 \gamma J} w^{2} \sigma_{m}^{2} \bar{u}^{2}(t) J_{w}^{2}+\frac{\alpha_{1}}{2 \gamma J} w^{2} \sigma^{2}\left(\pi_{1}(t)+\pi_{2}(t)\right)^{2} J_{w}^{2} \\
& \left.+\frac{\alpha_{1}}{2 \gamma J} b^{2}\left(w \pi_{1}(t) J_{w}+J_{x}\right)^{2}+\frac{\alpha_{1}}{2 \gamma J} b^{2}\left(w \pi_{2}(t) J_{w}-J_{x}\right)^{2}+\frac{\gamma J}{\alpha_{2}} \lambda\left(\phi^{*}-1\right)\right\}=0
\end{aligned}
$$

Differentiating Eq. (17) w.r.t. $q(t)$ implies

$$
J_{w}(1+\theta) \lambda \mu_{\infty}+\frac{1}{\alpha_{2}} \gamma J \lambda \frac{\partial \phi^{*}}{\partial q}=0
$$

According to (13) and (16), we know that the optimal reinsurance strategy $q^{*}(t)$ satisfies

$$
(1+\theta) \mu_{\infty}=\exp \left\{\frac{\alpha_{2}}{\gamma} E^{P}\left[e^{P(t) q^{*}(t) \gamma Y(t)}-1\right]\right\} E^{P}\left[Y(t) e^{P(t) q^{*}(t) \gamma Y(t)}\right]
$$

Thus, $q^{*}(t)>0$. If not, then we would have $e^{P(t) q^{*}(t) \gamma Y(t)}<1$ and

$$
\exp \left\{\frac{\alpha_{2}}{\gamma} E^{P}\left[e^{P(t) q^{*}(t) \gamma Y(t)}-1\right]\right\} E^{P}\left[Y(t) e^{P(t) q^{*}(t) \gamma Y(t)}\right]<E^{P}[Y(t)]<(1+\theta) \mu_{\infty},
$$

which would cause a contradiction with (18). According to the first-order condition for $\bar{u}, \pi_{1}, \pi_{2}$, we have

$$
\begin{aligned}
& J_{w} \mu_{m} w+J_{w w} w^{2} \sigma_{m}^{2} \bar{u}(t)+\frac{\alpha_{1}}{\gamma J} w^{2} \sigma_{m}^{2} J_{w}^{2} \bar{u}(t)=0 ; \\
& -J_{w} w x l_{1}+J_{w w} w^{2} \sigma^{2}\left(\pi_{1}(t)+\pi_{2}(t)\right)+J_{w w} w^{2} b^{2} \pi_{1}(t)+J_{w x} w b^{2} \\
& +\frac{\alpha_{1}}{\gamma J} w J_{w}\left[w J_{w} \sigma^{2}\left(\pi_{1}(t)+\pi_{2}(t)\right)+b^{2}\left(J_{x}+w \pi_{1}(t) J_{w}\right)\right]=0 ; \\
& J_{w} w x l_{2}+J_{w w} w^{2} \sigma^{2}\left(\pi_{1}(t)+\pi_{2}(t)\right)+J_{w w} w b^{2} \pi_{2}(t)-J_{w x} w b^{2} \\
& +\frac{\alpha_{1}}{\gamma J} w J_{w}\left[w J_{w} \sigma^{2}\left(\pi_{1}(t)+\pi_{2}(t)\right)+b^{2}\left(w \pi_{2}(t) J_{w}-J_{x}\right)\right]=0 ;
\end{aligned}
$$

Therefore, the optimal investment strategy is given by

$$
\begin{gathered}
\bar{u}^{*}(t)=-\frac{J_{w} w \mu_{m}}{w^{2} \sigma_{m}^{2}\left(J_{w w}+\frac{\alpha_{1} J_{w}^{2}}{\gamma J}\right)}=\frac{\mu_{m}}{w \sigma_{m}^{2} P(t)\left(\alpha_{1}+\gamma\right)}, \\
\pi_{1}^{*}(t) \quad=-\frac{1}{w\left(J_{w w}+\frac{\alpha_{1} J_{w}^{2}}{\gamma J}\right)}\left[J_{w x}+\frac{\alpha_{1} J_{x} J_{w}}{\gamma J}+\frac{1}{2}\left(\frac{l_{2}-l_{1}}{2 \sigma^{2}+b^{2}}-\frac{l_{1}+l_{2}}{b^{2}}\right) x J_{w}\right] \\
=-\frac{M(t) x}{w P(t)}+\left(\frac{l_{2}-l_{1}}{2 \sigma^{2}+b^{2}}-\frac{l_{1}+l_{2}}{b^{2}}\right) \frac{x}{2 w P(t)\left(\alpha_{1}+\gamma\right)}, \\
\pi_{2}^{*}(t) \quad=\frac{1}{w\left(J_{w w}+\frac{\alpha_{1} J_{w}^{2}}{\gamma J}\right)}\left[J_{w x}+\frac{\alpha_{1} J_{x} J_{w}}{\gamma J}-\frac{1}{2}\left(\frac{l_{2}-l_{1}}{2 \sigma^{2}+b^{2}}+\frac{l_{1}+l_{2}}{b^{2}}\right) x J_{w}\right] \\
=\frac{M(t) x}{w P(t)}+\left(\frac{l_{2}-l_{1}}{2 \sigma^{2}+b^{2}}+\frac{l_{1}+l_{2}}{b^{2}}\right) \frac{x}{2 w P(t)\left(\alpha_{1}+\gamma\right)} .
\end{gathered}
$$


Inserting $q^{*}(t)$ and $\bar{u}^{*}(t), \pi_{1}^{*}(t), \pi_{2}^{*}(t)$ into (17) yields

$$
\begin{aligned}
& -\gamma J\left[P^{\prime}(t) w+Q^{\prime}(t)+\frac{1}{2} M^{\prime}(t) x^{2}\right]-\gamma P(t) J\left[w r+\lambda \mu_{\infty}(\eta-\theta\right. \\
& \left.\left.+(1+\theta) q^{*}(t)\right)\right]+\left(l_{1}+l_{2}\right) M(t) x^{2} \gamma J+b^{2} \gamma M(t) J\left(\gamma M(t) x^{2}-1\right)+\frac{\gamma J}{\alpha_{2}} \lambda\left(\phi^{*}-1\right) \\
& +\alpha_{1} b^{2} \gamma J M^{2}(t) x^{2}-\frac{\mu_{m}^{2} \gamma J}{2 \sigma_{m}^{2}\left(\alpha_{1}+\gamma\right)}+\frac{\sigma^{2} x^{2}\left(l_{2}-l_{1}\right)^{2} \gamma}{2\left(2 \sigma^{2}+b^{2}\right)^{2}\left(\alpha_{1}+\gamma\right)}+\frac{\gamma J x^{2}}{\alpha_{1}+\gamma}\left[-\frac{b^{2}}{2} M^{2}(t)\left(\alpha_{1}+\gamma\right)^{2}\right. \\
& \left.-\lambda_{1}\left(\alpha_{1}+\gamma\right) M(t)+\frac{b^{2}}{8}\left(\frac{l_{2}-l_{1}}{2 \sigma^{2}+b^{2}}-\frac{l_{1}+l_{2}}{b^{2}}\right)^{2}+\frac{\lambda_{1}}{2}\left(\frac{l_{2}-l_{1}}{2 \sigma^{2}+b^{2}}-\frac{l_{1}+l_{2}}{b^{2}}\right)\right] \\
& +\frac{\gamma J x^{2}}{\alpha_{1}+\gamma}\left[-\frac{b^{2}}{2} M^{2}(t)\left(\alpha_{1}+\gamma\right)^{2}-\lambda_{2}\left(\alpha_{1}+\gamma\right) M(t)+\frac{b^{2}}{8}\left(\frac{l_{2}-l_{1}}{2 \sigma^{2}+b^{2}}+\frac{l_{1}+l_{2}}{b^{2}}\right)^{2}\right. \\
& \left.-\frac{\lambda_{2}}{2}\left(\frac{l_{2}-l_{1}}{2 \sigma^{2}+b^{2}}+\frac{l_{1}+l_{2}}{b^{2}}\right)\right]=0
\end{aligned}
$$

Letting the coefficients of $w$ and $x^{2}$ be zero, we get

$$
\begin{gathered}
-\gamma P^{\prime}(t)-\gamma P(t) r=0 \\
M^{\prime}(t)+\frac{1}{2\left(\alpha_{1}+\gamma\right)}\left(\frac{\left(l_{2}-l_{1}\right)^{2}}{2 \sigma^{2}+b^{2}}+\frac{\left(l_{1}+l_{2}\right)^{2}}{b^{2}}\right)=0 \\
-\gamma Q^{\prime}(t)-\gamma P(t)\left(\eta-\theta+(1+\theta) q^{*}(t)\right) \lambda \mu_{\infty}-b^{2} \gamma M(t)+\frac{1}{\alpha_{2}} \gamma \lambda\left(\phi^{*}-1\right) \\
-\frac{\mu_{m}^{2}}{2 \sigma_{m}^{2}} \frac{\gamma}{\alpha_{1}+\gamma}=0 .
\end{gathered}
$$

Taking into account the boundary conditions $P(T)=1, M(T)=0$ and $Q(T)=0$, we know that

$$
\begin{aligned}
& P(t)=e^{r(T-t)}, M(t)=\frac{k(T-t)}{\alpha_{1}+\gamma}, \\
& Q(t)=\left(\frac{\mu_{m}^{2}}{2 \sigma_{m}^{2}\left(\alpha_{1}+\gamma\right)}+\frac{\lambda}{\alpha_{2}}\right)(T-t)+\frac{b^{2} k(T-t)^{2}}{2\left(\alpha_{1}+\gamma\right)}-\frac{\left(1-e^{r(T-t)}\right)(\eta-\theta) \lambda \mu_{\infty}}{r} \\
& +(1+\theta) \lambda \mu_{\infty} \int_{t}^{T} e^{r(T-t)} q^{*}(t) d t-\frac{\lambda}{\alpha_{2}} \int_{t}^{T} \exp \left\{\frac{\alpha_{2}}{\gamma} E^{P}\left[e^{e^{r(T-t)} q^{*}(t) \gamma Y(t)}-1\right]\right\} d t,
\end{aligned}
$$

where $k=\frac{1}{2}\left(\frac{\left(l_{2}-l_{1}\right)^{2}}{2 \sigma^{2}+b^{2}}+\frac{\left(l_{1}+l_{2}\right)^{2}}{b^{2}}\right)$. Therefore, the optimal investment strategy now takes the simpler form

$$
\begin{aligned}
& \pi_{m}^{*}(t)=\frac{e^{-r(T-t)}}{w\left(\alpha_{1}+\gamma\right)}\left(\frac{\mu_{m}}{\sigma_{m}^{2}}-\frac{\beta\left(l_{2}-l_{1}\right) x}{b^{2}+2 \sigma^{2}}\right), \\
& \pi_{1}^{*}(t)=\frac{e^{-r(T-t)} x}{w\left(\alpha_{1}+\gamma\right)}\left[\frac{1}{2}\left(\frac{l_{2}-l_{1}}{b^{2}+2 \sigma^{2}}-\frac{l_{1}+l_{2}}{b^{2}}\right)-k(T-t)\right], \\
& \pi_{2}^{*}(t)=\frac{e^{-r(T-t)} x}{w\left(\alpha_{1}+\gamma\right)}\left[\frac{1}{2}\left(\frac{l_{2}-l_{1}}{b^{2}+2 \sigma^{2}}+\frac{l_{1}+l_{2}}{b^{2}}\right)+k(T-t)\right] .
\end{aligned}
$$

Summarizing the discussion above and using a verification theorem whose proof is straightforward (see Yi et al. (2015) and Mataramvura and Øksendal (2008)), we have the following Theorem.

Theorem 4.1. For the optimization problem (9), the value function is given by

$$
J(t, w, x)=-\frac{\delta}{\gamma} \exp \left\{\left\{-\gamma\left[P(t) w+Q(t)+\frac{1}{2} M(t) x^{2}\right]\right\}\right.
$$

where $P(t), Q(t)$ and $M(t)$ are given by (25), and the corresponding optimal reinsurance- 
investment strategy is given by

$$
\pi^{*}=\left(q^{*}(t), \pi_{m}^{*}(t), \pi_{1}^{*}(t), \pi_{2}^{*}(t)\right)
$$

with $q^{*}(t)$ determined by equation (18), and $\pi_{m}^{*}(t), \pi_{1}^{*}(t), \pi_{2}^{*}(t)$ are given in (26).

The optimal reinsurance strategy is dependent on both the ambiguity aversion level $\alpha_{2}$ and the absolute risk aversion coefficient $\gamma$, but does not involve the market and stock parameters. The optimal investment strategy is driven by the sum $\alpha_{1}+\gamma$ and depends on the mispricing level $x$. In particular, our formula $\pi^{*}$ shows that, as long as $l_{1}=l_{2}$ the optimal strategy invested in both stocks is a L-S strategy which can be used as long-term arbitrage opportunity, whereas the insurer can make use of temporary diversification to make profits when $l_{1} \neq l_{2}$.

\subsection{Special cases}

This section is devoted to seeking optimal value functions and optimal strategies for the ANI, who ignores ambiguity aversion, and for the AAI who ignores the jump term and mispricing in the model. Moreover, the investment-only problem is discussed to show the importance of reinsurance for AAI in the optimization decision.

(I) Case 1: (No ambiguity aversion) In this case we discuss two conditions. If there are no drift distortions (i.e $\varphi \equiv 0)$ and no claim intensity distortion $(\phi \equiv 0)$ and all the ambiguity-aversion coefficients equal 0, i.e., $\alpha_{1}=\alpha_{2}=0$ in our model, the optimization problem (9) for AAI reduces to an optimization problem for ANI. The surplus of the ANI under the initial measure $P$ is given by Eq.(5) and the optimization problem is

$$
J_{0}(t, w, x)=\max _{\pi_{0} \in \Pi_{0}} E_{0}^{P}\left[-\frac{1}{\gamma} \exp \left\{-\gamma W^{\pi}(T)\right]\right.
$$

With similar method to Section 4.1, we can derive the optimal strategy and optimal value function for the ANI.

Proposition 4.2. For optimal problem (27), the value function for ANI is given by

$$
J_{0}(t, w, x)=-\frac{\delta}{\gamma} \exp \left\{-\gamma\left(P(t) w+Q_{0}(t)+\frac{1}{2} M_{0}(t) x^{2}\right)\right\}
$$


where

$$
\begin{aligned}
& P_{0}(t)=P(t)=\exp \{r(T-t)\} \\
& Q_{0}(t)=\frac{\mu_{m}^{2}}{2 \sigma_{m}^{2} \gamma}(T-t)+\frac{b^{2} k(T-t)^{2}}{2 \gamma}-\frac{\left(1-e^{r(T-t)}\right)(\eta-\theta) \lambda \mu_{\infty}}{r} \\
& +(1+\theta) \lambda \mu_{\infty} \int_{t}^{T} e^{r(T-t)} q_{0}^{*}(t) d t-\frac{\lambda}{\gamma} \int_{t}^{T} E\left[\exp \left\{P(t) q_{0}^{*}(t) \gamma Y\right\}-1\right] d t ; \\
& M_{0}(t)=\frac{k(T-t)}{\gamma}
\end{aligned}
$$

the corresponding optimal investment strategy $\pi_{0}^{*}=\left.\pi^{*}\right|_{\alpha_{1}=\alpha_{2}=0}$, where $\pi^{*}$ is given by Theorem 4.1 and the optimal reinsurance strategy $q_{0}^{*}(t)$ solves the following equation

$$
(1+\theta) E^{P}[Y(t)]=E^{P}\left[Y(t) e^{P(t) q_{0}^{*}(t) \gamma Y(t)}\right]
$$

We find that our optimal reinsurance-investment strategy for the ANI in Proposition 4.2 can be reduced to the result of the Xu et al. (2008), where they do not consider the market index or the mispricing. Our optimal reinsurance strategy for the ANI is same as that of Xu et al. (2008).

Next, we discuss another condition: there exist a drift distortion (i.e $\varphi=\left(h_{m}, 0, h_{1}, h_{2}\right), h_{1}=$ $-a x, h_{2}=a x$ and $a$ is constant) and a claim intensity distortion $\phi$. Here we assume that there exists a alternative measure $Q_{0}$, which is different from the worst case measure $Q^{*}$ in the Section 4.1 and the reference measure $P$. The idea here is that an insurer may mistakenly use $P$ when in fact the correct measure is $Q_{0}$, and may unwisely ignore ambiguity. Under the measure $Q_{0}$ and no ambiguity aversion $\alpha_{1}=\alpha_{2}=0$, we calculate the expected utility with the optimal strategy $\pi_{P}^{*}=\left(\pi_{0}^{*}, q_{0}^{*}\right)$ given in the Proposition 4.2 , assuming that the insurer mistakenly believes $P$ is correct. To simplify the exposition, we assume identical liquidity parameters $l_{1}=l_{2}$. The value function would then be defined as

$$
J_{0 r}(t, w, x):=E_{t, w, x}^{Q_{0}}\left\{-\frac{\delta}{\gamma} e^{-\gamma w_{\pi_{P}^{*}}(T)}\right\}
$$

where the wealth process of the ANI with investment strategy $\pi_{P}^{*}$ under measure $Q_{0}$ is given 
by

$$
\begin{aligned}
& d W_{\pi_{P}^{*}}=\left(r W_{\pi_{P}^{*}}+\left(\eta-\theta+(1+\theta) q_{0}^{*}\right) \lambda \mu_{\infty}\right) d t+\frac{\mu_{m}\left(\mu_{m}-\sigma_{m} h_{m}\right)}{\sigma_{m}^{2} \gamma} e^{-r(T-t)} d t \\
& +X(t)^{2} e^{-r(T-t)} \frac{\left(l_{1}+l_{2}\right)^{2}(T-t)\left(l_{1}+l_{2}-2 a b\right)+\left(l_{1}+l_{2}\right)^{2}-2 a b\left(l_{1}+l_{2}\right)}{2 b^{2} \gamma} d t \\
& +\frac{\mu_{m}}{\sigma_{m} \gamma} e^{-r(T-t)} d B_{t}^{Q_{0}}+X(t) \frac{l_{1}+l_{2}}{2 b \gamma} e^{-r(T-t)}\left(1+\left(l_{1}+l_{2}\right)(T-t)\right)\left(d Z_{2 t}^{Q_{0}}-d Z_{1 t}^{Q_{0}}\right) \\
& -q_{0}^{*} \int_{R^{+}} y(\tilde{N}(d t, d y)+\phi \lambda d F(y) d t) .
\end{aligned}
$$

The use of the incorrect model $P$ can have dramatic consequences if it is not sufficiently close to $Q_{0}$. For example we have the following proposition; a more detailed analysis of its conditions is in Section 5.

Proposition 4.3. Let $\ell=l_{1}+l_{2}$. If

$$
2 a b>\ell
$$

and

$$
(2 a b-\ell)\left(T+\ell^{-1}\right)\left[1+\frac{1}{\ell(T-t)}\left(e^{-\ell T}\left(e^{\ell t}-e^{-\ell t}\right)-1-2^{-1}\left(e^{-2 \ell t}+e^{-2 \ell T}\right)\right)\right] \geq \frac{1}{2}
$$

then

$$
J_{0 r}(t, w, x)=-\infty
$$

Proof: We sketch the proof of this proposition, beginning with a discussion of where the condition $2 a b>\ell$ comes from, using the shorthand notation $\ell=l_{1}+l_{2}$ as in the statement of the proposition. In the dynamics of the process $W_{\pi_{P}^{*}}$ above, we note that the third term (line 31) is of the form $K(t) X(t)^{2}$, where

$$
K(t)=e^{-r(T-t)} \frac{\ell^{2}(T-t)(\ell-2 a b)+\ell^{2}-2 a b \ell}{2 b^{2} \gamma} .
$$

To get an infinite value function, it is preferable that this constant $K(t)$ be negative, which implies that

$$
\ell+(T-t)(\ell-2 a b) \ell<2 a b .
$$

It is easy to check that this last condition also implies that $\ell<2 a b$. In any case this condition is an assumption of the proposition.

In particular, $\ell<2 a b$ implies that one can get a lower bound on $|K(t)|$ by setting $t=0$ :

$$
|K(0)| \leq|K(t)| .
$$


Another important point is that under conditions by which the integral over $[t, T]$ of the term in line (31) has infinite exponential moments, the integral of the second term in line (32) also has infinite exponential moments, while all the terms in lines (30) and (33) have finite such moment. Thus we only concentrate on checking that the value function $J_{0 r 2}$ of the term corresponding to line (31) is infinite. Now, thanks to the inequalities $\ell<2 a b$ and $|K(0)| \leq|K(t)|$ discussed above, and also using Jensen's inequality, we write

$$
\begin{aligned}
J_{0 r 2}(t, w, x) & :=\frac{-\delta}{\gamma} \mathbf{E}\left[\exp \left(-\gamma \int_{t}^{T} K(s) X^{2}(s) d s\right)\right] \\
& =\frac{-\delta}{\gamma} \mathbf{E}\left[\exp \left(\gamma \int_{t}^{T}|K(s)| X^{2}(s) d s\right)\right] \\
& \leq \frac{-\delta}{\gamma} \mathbf{E}\left[\exp \left(\gamma|K(0)| \int_{t}^{T} X^{2}(s) d s\right)\right] \\
\leq & \frac{-\delta}{\gamma} \mathbf{E}\left[\exp \left(\gamma|K(0)| \frac{1}{T-t}\left(\int_{t}^{T} X(s) d s\right)^{2}\right)\right]
\end{aligned}
$$

Since $\int_{t}^{T} X(s) d s$ is a Gaussian variable, it is elementary that the last expectation above is infinite if and only if the variance of that random variable is large enough; specifically in our case, we evidently only need

$$
\gamma|K(0)| \frac{1}{T-t} \operatorname{Var}\left(\int_{t}^{T} X(s) d s\right) \geq \frac{1}{2}
$$

After some computation, we find

$$
\begin{aligned}
\operatorname{Var}\left(\int_{t}^{T} X(s) d s\right) & =2 \int_{t}^{T}\left(\int_{t}^{s} \mathbf{E}[X(s) X(r)] d r\right) d s \\
& =2 \int_{t}^{T}\left(\int_{t}^{s} \frac{b^{2}}{\ell}\left(e^{-\ell(s-t)}-e^{-\ell(s+t)}\right) d r\right) d s \\
& =\frac{2 b^{2}}{\ell^{2}}\left[T-t+\frac{1}{\ell}\left(e^{-\ell T}\left(e^{\ell t}-e^{-\ell t}\right)-1-2^{-1}\left(e^{-2 \ell t}+e^{-2 \ell T}\right)\right)\right] .
\end{aligned}
$$

Therefore, condition (34) becomes

$$
(2 a b-\ell)\left(T+\ell^{-1}\right)\left[1+\frac{1}{\ell(T-t)}\left(e^{-\ell T}\left(e^{\ell t}-e^{-\ell t}\right)-1-2^{-1}\left(e^{-2 \ell t}+e^{-2 \ell T}\right)\right)\right] \geq \frac{1}{2}
$$

as announced.

(II) Case 2: (No jumps) We consider the optimal reinsurance-investment problem for an AAI without a jump term in the insurance model. Here we approximate the surplus process (1) of the insurer via a diffusion. Specifically, then there exists a standard Brownian 
motion $\left\{B_{0 t}^{Q}\right\}$ under measure $Q$ and with reinsurance strategy $\left\{q_{1}(t)\right\}$; the surplus follows a diffusion process (see Grandell (1991) and Gu et al. (2012)):

$$
d R(t)=\left[(\eta-\theta) \lambda \mu_{\infty}+\theta q_{1}(t) \lambda \mu_{\infty}\right] d t+\sqrt{\lambda} q_{1}(t) \sigma_{\infty} d B_{0 t}^{Q}
$$

Therefore, the AAI's wealth process with investment strategy $\left(\pi_{m}^{1}(t), \pi_{1}^{1}(t), \pi_{2}^{1}(t)\right)$ under measure $Q$ can be described by

$$
\begin{aligned}
d W(t)= & {\left[(\eta-\theta) \lambda \mu_{\infty}+\theta q_{1}(t) \lambda \mu_{\infty}\right] d t+\sqrt{\lambda} q_{1}(t) \sigma_{\infty} d B_{0 t}^{Q}+W(t)\left[r+\mu_{m} \bar{u}^{1}(t)\right.} \\
& +x\left(\pi_{2}^{1}(t) l_{2}-\pi_{1}^{1}(t) l_{1}\right)-h_{m}(t) \sigma_{m} \bar{u}^{1}(t)-\sigma h(t)\left(\pi_{1}^{1}(t)+\pi_{2}^{1}(t)\right) \\
& \left.-b\left(\pi_{1}^{1}(t) h_{1}(t)+\pi_{2}^{1}(t) h_{2}(t)\right)\right] d t+W(t)\left[\sigma_{m} \bar{u}^{1}(t) d B_{t}^{Q}+\sigma\left(\pi_{1}(t)+\pi_{2}(t)\right) d Z_{t}^{Q}\right] \\
& +b W(t)\left(\pi_{1}(t) d Z_{1 t}^{Q}+\pi_{2}(t) d Z_{2 t}^{Q}\right)
\end{aligned}
$$

where $\bar{u}^{1}(t)=\pi_{m}^{1}(t)+\beta\left(\pi_{1}^{1}(t)+\pi_{2}^{1}(t)\right)$ and $\left\{B_{0 t}^{Q}\right\}, \quad\left\{B_{t}^{Q}\right\}, \quad\left\{Z_{1 t}^{Q}\right\}, \quad\left\{Z_{2 t}^{Q}\right\}$ and $\left\{Z_{t}^{Q}\right\}$ are independent each other. The corresponding optimization problem is

$$
J^{1}(t, w, x)=\sup _{\pi^{1} \in \Pi^{1}} \inf _{Q \in \mathcal{Q}} E_{t, w, x}^{Q}\left\{-\frac{\delta}{\gamma} e^{-\gamma w(T)}+\int_{t}^{T} \frac{R_{1}(s)}{\psi_{1}(s)} d s\right\}
$$

With similar methods, the optimal reinsurance-investment strategy $\pi^{1^{*}}$ is given by

$$
\begin{aligned}
q^{1^{*}}(t) & =\frac{\theta \mu_{\infty}}{\gamma} e^{r(T-t)}, \\
\pi_{m}^{1 *}(t) & =\frac{e^{-r(T-t)}}{w\left(\alpha_{1}+\gamma\right)}\left(\frac{\mu_{m}}{\sigma_{m}^{2}}-\frac{\beta\left(l_{2}-l_{1}\right) x}{b^{2}+2 \sigma^{2}}\right), \\
\pi_{1}^{1^{*}}(t) & =\frac{e^{-r(T-t)} x}{w\left(\alpha_{1}+\gamma\right)}\left[\frac{1}{2}\left(\frac{l_{2}-l_{1}}{b^{2}+2 \sigma^{2}}-\frac{l_{1}+l_{2}}{b^{2}}\right)-k(T-t)\right], \\
\pi_{2}^{1^{*}}(t) & =\frac{e^{-r(T-t)} x}{w\left(\alpha_{1}+\gamma\right)}\left[\frac{1}{2}\left(\frac{l_{2}-l_{1}}{b^{2}+2 \sigma^{2}}+\frac{l_{1}+l_{2}}{b^{2}}\right)+k(T-t)\right],
\end{aligned}
$$

and the optimal value function is

$$
J^{1}(t, x, w)=-\frac{1}{\gamma} \exp \left\{-\gamma\left[P(t) w+Q^{1}(t)+\frac{1}{2} M(t) x^{2}\right]\right\}
$$

where $P(t)$ and $M(t)$ are given by Theorem 4.1 and

$$
Q^{1}(t)=\frac{b^{2} k(T-t)^{2}}{2\left(\alpha_{1}+\gamma\right)}-\frac{\left(1-e^{r(T-t)}\right)(\eta-\theta) \lambda \mu_{\infty}}{r}+\left(\frac{\theta^{2} \mu_{\infty}^{2} \lambda}{2 \sigma_{\infty}^{2} \gamma}+\frac{\mu_{m}^{2}}{2\left(\alpha_{1}+\gamma\right) \sigma_{m}^{2}}\right)(T-t)
$$

Obviously, in this case the ambiguity only affects the investment strategy. This can be compared to the diffusion model for an insurer in Yi et al. (2013) who discussed the optimal 
reinsurance-investment problem without mispricing.

(iii) Case 3: (No mispricing) If there are no mispricing opportuities in the market, i.e., $X(t) \equiv 0$, we assume that the AAI would purchase reinsurance and invest only in a market index and risk-free asset, and denote the optimal strategy by $\hat{\pi}=\left\{\left(\hat{q}(t), \hat{\pi}_{m}(t)\right)\right\}_{t \in[0, T]}$. In this case, we can seek the measure $\hat{Q}$ and strategy $\hat{\pi}$ to solve the optimization problem

$$
\hat{J}(t, w)=\sup _{\hat{\pi} \in \hat{\Pi}} \inf _{\hat{Q} \in \mathcal{Q}} E_{t, w, x}^{\hat{Q}}\left\{-\frac{\delta}{\gamma} e^{-\gamma w(T)}+\int_{t}^{T}\left(\frac{R_{1}(s)}{\psi_{1}^{\hat{\pi}}(s)}+\frac{R_{2}(s)}{\psi_{2}^{\hat{\pi}}(s)}\right) d s\right\}
$$

where $R_{1}(s)=\frac{1}{2} h_{m}^{2}(s)$ and $R_{2}(s)=\lambda(\phi(s) \ln \phi(s)-\phi(s)+1)$ measure the relative entropy arising from uncertainty, and $\psi_{1}^{\hat{\pi}}(s)=-\frac{\alpha_{1}}{\gamma \hat{J}(t, w, x)}$ and $\psi_{2}^{\hat{\pi}}(s)=-\frac{\alpha_{2}}{\gamma \hat{J}(t, w, x)}$ are the preference parameter with strategy $\hat{\pi}$, representing the ambiguity-averse preferences.

In the following, we use the similar method to Section 4.1 to solve (35). The corresponding HJB equation for (35) is given by

$$
\begin{aligned}
& 0=\sup _{\hat{\pi} \in \hat{\Pi}} \inf _{\hat{Q} \in \mathcal{Q}}\left\{\hat{J}_{t}+\hat{J}_{w}(\eta-\theta+(1+\theta) \hat{q}(t)) \lambda \mu_{\infty}+\hat{J}_{w} w\left(r+\mu_{m} \hat{\pi}_{m}(t)-\hat{\pi}_{m}(t) \mu_{m} h_{m}(t)\right)\right. \\
& +\frac{1}{2} J_{w w} w^{2} \sigma_{m}^{2} \hat{\pi}_{m}^{2}(t)+\phi(t) \lambda E^{\hat{Q}}[\hat{J}(t, w-\hat{q}(t) Y)-\hat{J}(t, w)]-\frac{\gamma \hat{J}}{2 \alpha_{1}} h_{m}^{2}(t)-\frac{\gamma \hat{J} \lambda}{\alpha_{2}}(\phi(t) \ln \phi(t) \\
& -\phi(t)+1)\},
\end{aligned}
$$

with boundary condition $\hat{P}(T)=1, \hat{Q}(T)=0$. Therefore, we guess that the value function $\hat{J}$ has the form

$$
\hat{J}(t, w)=-\frac{1}{\gamma} \exp \{-\gamma[\hat{P}(t) w+\hat{Q}(t)]\}
$$

After simple calculation, we obtain that

$$
\begin{gathered}
\hat{P}(t)=e^{r(T-t)}=P(t), \\
\hat{Q}(t)=\int_{t}^{T} e^{r(T-t)}\left(\eta-\theta+(1+\theta) q^{*}(t)\right) \lambda \mu_{\infty} d t-\frac{\lambda}{\alpha_{2}} \int_{t}^{T}\left(\phi^{*}-1\right) d t+\frac{\mu_{m}^{2}(T-t)}{2 \sigma_{m}^{2}\left(\alpha_{1}+\gamma\right)},
\end{gathered}
$$

and the optimal strategy is

$$
\hat{\pi}(t)=\left(\hat{q}^{*}(t), \hat{\pi}_{m}^{*}(t)\right)=\left(q^{*}(t),\left.\pi_{m}^{*}(t)\right|_{x=0}\right)
$$

where $q^{*}(t)$ and $\pi_{m}^{*}(t)$ are given by Theorem 4.1 .

Proposition 4.4. For optimization problem (35) without mispricing, the value function is given by

$$
\hat{J}(t, w)=-\frac{1}{\gamma} \exp \{-\gamma[P(t) w+\hat{Q}(t)]\}
$$


where $P(t)$ and $\hat{Q}(t)$ are given by (25) and (36), respectively. The corresponding optimal strategy $\hat{\pi}^{*}(t)$ is given by (37).

Again, this result can be compared to Yi et al.(2013) who discuss the optimal robust reinsurance-investment problem without mispricing in the framework of maximizing the expected utility, where their insurance model is described by a diffusion.

Remark 4.1. The value function without mispricing is less than value function with mispricing. By comparing two value functions, we can show the impact of mispricing investment opportunities on the optimal value function. This analysis will be presented in Section 5.

(iv) Case 4: (No reinsurance) If the insurer only invests in the financial market, and does not purchase reinsurance, the optimization problem (9) degenerates to investment-only problem. With $q^{*}(t) \equiv 1$ in Theorem 4.1, we easily derive the following Proposition

Proposition 4.5. For optimization problem (9), if only investment is discussed, the value function is given by

$$
\tilde{J}(t, w, x)=-\frac{1}{\gamma} \exp \left\{-\gamma\left[P(t) w+\tilde{Q}(t)+\frac{1}{2} M(t) x^{2}\right]\right\}
$$

where $P(t), M(t)$ are given by (25) and

$$
\begin{aligned}
\tilde{Q}(t)=\quad & \left(\frac{\mu_{m}^{2}}{2 \sigma_{m}^{2}\left(\alpha_{1}+\gamma\right)}+\frac{1}{\alpha_{2}}\right)(T-t)+\frac{b^{2} k(T-t)^{2}}{2\left(\alpha_{1}+\gamma\right)}-\frac{\left(1-e^{r(T-t)}\right)(\eta+1) \lambda \mu_{\infty}}{r} \\
& -\frac{\lambda}{\alpha_{2}} \int_{t}^{T} \exp \left\{\frac{\alpha_{2}}{\gamma} E^{P}\left[e^{e^{r(T-t)} \gamma Y(t)}-1\right]\right\} d t .
\end{aligned}
$$

and the corresponding optimal investment strategy is given by (26), i.e.,

$$
\tilde{\pi}^{*}=\left(\pi_{m}^{*}(t), \pi_{1}^{*}(t), \pi_{2}^{*}(t)\right)
$$

\section{Analysis of the results and numerical illustration}

In this section, we are interested in providing some numerical examples to show the impact of ambiguity aversion, mispricing and some parameters on the AAI's optimal strategy and optimal value function. We choose to work with parameters which are calibrated to the financial market. We use the calibration against the data derived by four Chinese bank stocks traded in both China A shares and Hong Kong H shares, found in table 1 of Yi et al. (2015). Thus throughout this section, unless otherwise stated, the basic parameters of the financial market are given by $r=0.03, \gamma=0.8, \sigma=0.3, \quad b=0.3, \lambda=10, l_{1}=l_{2}=0.5$; 
the other parameters are taken as $\eta=0.1, \theta=0.2, T=4, \beta=1.1, w=2, \alpha_{1}=\alpha_{2}=0.4$. In addition, we assume that the claim size $Y_{i}$ follows the exponential distribution with parameter $\lambda_{Y}=6$, and therefore the claim size density function is $f(y)=6 e^{-6 y}$.

\subsection{Analysis of optimal strategy}

In this subsection, we study the impact of ambiguity aversion levels $\alpha_{1}, \alpha_{2}$ and pricing error $X$ on the optimal investment and reinsurance strategies. As shown in Theorem 4.1, ambiguity aversion level $\alpha_{1}$ only has an effect on the optimal investment strategy, but no effect on the optimal reinsurance strategy. Similarly, ambiguity aversion $\alpha_{2}$ only has an effect on the optimal reinsurance strategy. The reason comes from formula (26): the allocations for market index and risky asset positions do not depend on the parameters from the insurance model, i.e. $\lambda, \theta, \eta$. The same occurs for the reinsurance strategy $q$, which does not depend on the risky asset and market parameters: the pricing error of risky assets does not affect the optimal reinsurance strategy. This separation of the impact of aversion parameters justifies their interpretation as parameters affecting modeling uncertainty for each of the two components of our model. It is due to the dual structure of our homothetic robustness penalization function. This greatly facilitates the quantitative analysis. Being able to exhibit this separation is the only consistent way of interpreting the AAI's two sources of modeling uncertainty (rates of return in the financial market, and insurance claims intensity).

Now, we discuss the impact of ambiguity aversion and pricing error on the optimal investment strategy. Let us first discuss the effect of changing model ambiguity. A naive intuition might indicate that an increase in ambiguity aversion on the mispricing and market models (increase in $\alpha_{1}$ ) would have the AAI worry about taking advantage of the risky markets, and thus perhaps decrease her holdings in risky assets, for instance. Instead, we see in the expression of $\pi_{m}^{*}$ in (26) that this is only true either when $l_{2}-l_{1}$ and the mispricing level $x$ are of opposite signs, or if $|x|$ is sufficiently small. If instead $l_{2}-l_{1}$ and the mispricing level $x$ are of the same signs, if $x$ sufficiently large, then the effect of ambiguity is reversed on the position in market risk. To make this clearer, fix ideas by assuming that $x>0$ and $l_{2}-l_{1}>0$. This means that $P_{1}$ is overpriced relative to $P_{2}$ and the rate of reversion of the underpriced stock $P_{2}$ back to the mean is faster than the rate of reversion of the overpriced stock back to the mean. Thus an advantage conferred by the mispricing which one would exploit by having a large long position in the underpriced stock $P_{2}$, will have a tendency to disappear faster than if the two rates $l_{1}$ and $l_{2}$ were identical. In this situation, by the first line in (26), it turns out that if the mispricing $x$ is large enough, it will be to the AAI's advantage to invest more in market risk when ambiguity increases. This says that the AAI, who worries about the models for the two stocks and the market, appears to be more concerned about the rate at which she might lose her mispricing advantage on the long side, than about the market model being wrong. As we said, by (26), this only occurs when 
$x$ is large enough, specifically, when it exceeds the threshold $\frac{b^{2}+2 \sigma^{2}}{\sigma_{m}^{2}} \frac{\mu_{m}}{\beta\left(l_{2}-l_{1}\right)}$. Evidently, this threshold depends explicitly on the ratio of stock volatility to market volatility: when it is low, the threshold is low. In conclusion, in the case of mispriced stocks where long positions are more vulnerable over time than short ones, an ANI may take greater refuge in market risk when her risk ambiguity is raised if mispricing is high, and more often when market volatility is relatively high. The general picture is similarly complex from an analytical standpoint. We now provide further, more quantitative details based on numerical investigation of the formulas in (26).

When $l_{1}=l_{2}$, the mispricing $X$ has no impact on the proportion $\pi_{m}^{*}$ invested in market index, and ambiguity aversion $\alpha_{1}$ has a negative relationship with $\pi_{m}^{*}(t)$, i.e., $\pi_{m}^{*}(t)$ will decrease with the increase of aversion to financial model ambiguity. This is in line with common-sense intuition of a risk-averse investor who decreases her risky position in order to reduce risk, and we see here that the intuition extends to modeling risk. As shown in (26), the optimal strategy invested in the pair of risky assets is an L-S strategy. That is to say, $\pi_{1}^{*}(t)=-\pi_{2}^{*}(t)$, the insurer exploits the pricing error to simultaneously buy underpriced stock 2 and sell (or short) overpriced stock 1 with equal size, see Liu and Longstaff (2004) and Jurek and Yang (2006). It is obvious from (26) that $\pi_{1}^{*}(t)$ decreases with $x$ and increases with the change of ambiguity aversion $\alpha_{1}$. On the contrary, $\pi_{2}^{*}(t)$ increases with $x$ and decreases with $\alpha_{1}$. The sensitivity of $\pi_{1}^{*}(t)$ and $\pi_{2}^{*}(t)$ about $x$ and $\alpha_{1}$ are quantified in Figure 1(a) fixing $t=2$. Including in this figure is the case where $\alpha_{1}=0$, when the insurer is an ANI who believe the market model completely. Quantitatively, Figure 1(a) tells us the ANI tends to invest more, but not much more than the AAI does.

Next, we look at the effect of changing levels of mispricing, in the asymmetric reversion rate case. Without loss of generality as we did in the previous illustration, we assume that $l_{1}<l_{2}$. It is obvious that $\pi_{m}^{*}(t)$ and $\pi_{1}^{*}(t)$ decrease with mispricing and $\pi_{2}^{*}(t)$ increases with mispricing. Figure 1(c) shows that the AAI would short stock 1 and hold stock 2 when $x>0$, which is intuitively clear as mentioned in the above analysis of sensitivity with respect to $\alpha_{1}$. Unlike in the L-S strategy, the difference of the absolute values of the positions in the two stock is not zero, and we also note that it increases with time. Specifically, notice that we are increasing the intensity of the effect of misprincing on $P_{2}$; this means, by the original model for $P_{2}$, and the fact that $x>0$, that $P_{2}$ will have a tendency to increase back to a value closer to $P_{1}$ faster than the same effect on $P_{1}$. In other words, the mispricing would tend to disappear faster than in the case described in Figure 1(a), because of a stronger mispricing mean-reversion effect on $P_{2}$. One may then wonder whether one should increase or decrease the long position compared to the short one; Figure 1(c) shows that one should increase the long position, more than one should increase (the absolute value of) the short position. The presence of the positive term $k(T-t)$ in the formula for $\pi_{2}^{*}$ in (26) shows that one should increase the long position even more for longer time horizons. This can be 


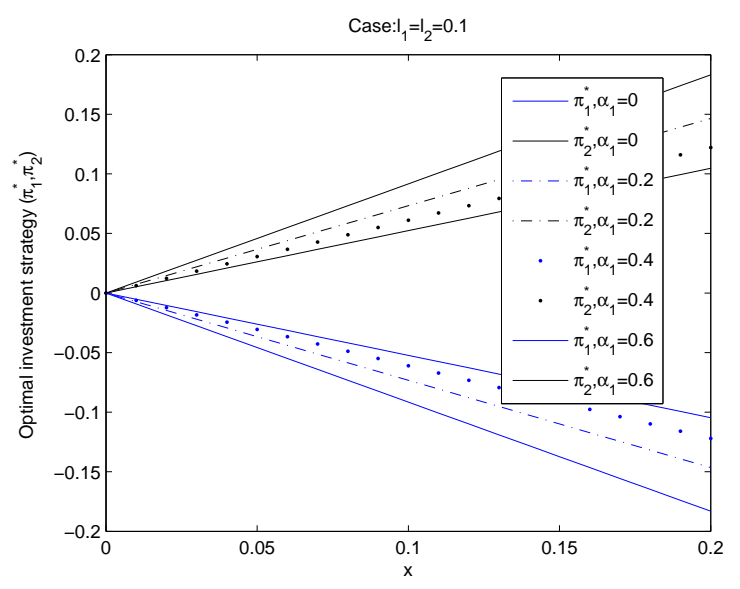

(a)

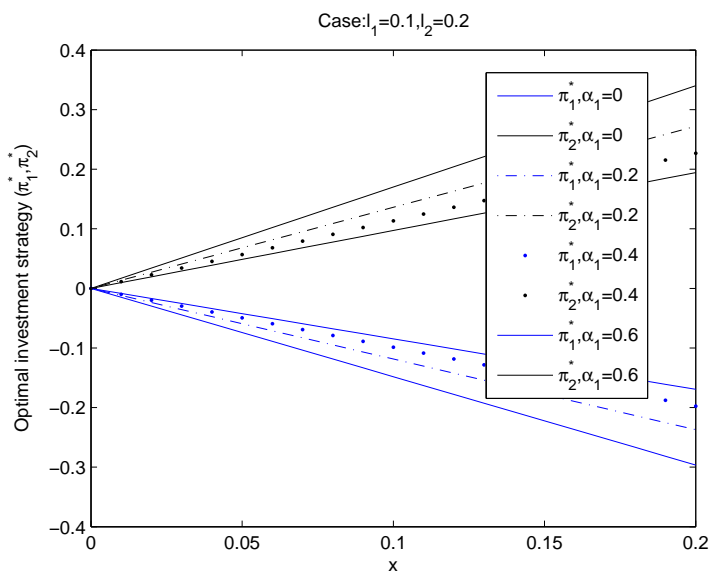

(c)

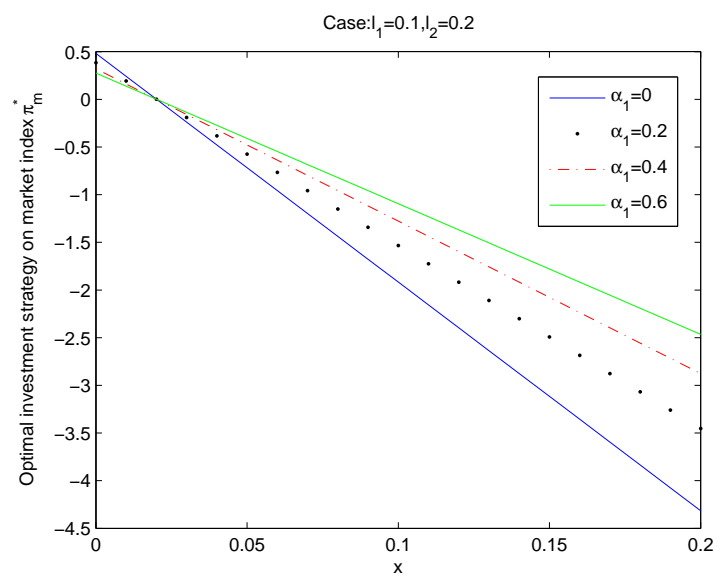

(b)

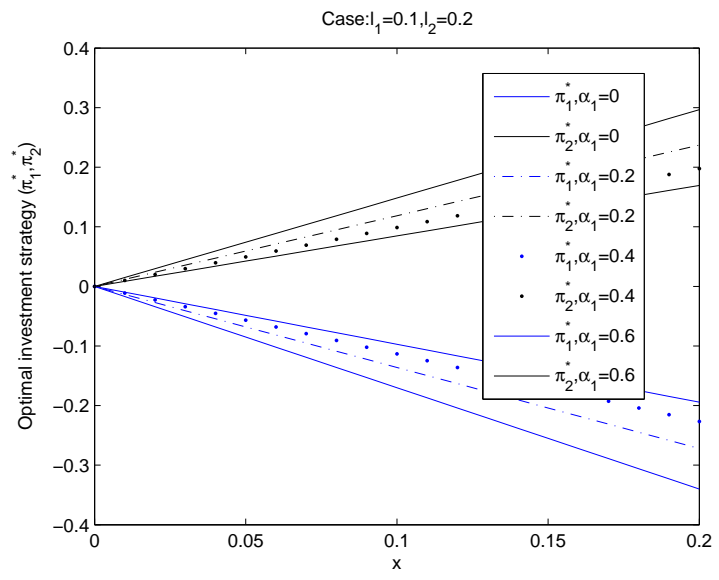

(d)

Figure 1: Impact of $\alpha_{1}$ and of $x$ on the optimal investment strategy

interpreted as saying that the AAI is trying to take advantage of stronger mispricing today, as opposed to hoping that the mispricing will persist into the future. The case for $l_{1}>l_{2}$ is illustrated in Figure 1 (d), and is symmetric to the opposite case.

In the following, we discuss the impact of ambiguity aversion on the optimal reinsurance strategy. Recall that the mispricing does not affect the reinsurance strategy. In contrast with the analysis of the expressions in (26) which are explicit and straightforward, it is much more difficult to analyze the formula for $q^{*}$ since it is given in (18) via the solution of an equation involving transcendental functions. Thus we must rely entirely on numerical analyses of this equation's solution for various sets of parameters. However, interestingly, the impact on $q^{*}$ is much simpler to describe than what we saw regarding $\pi_{1}^{*}, \pi_{2}^{*}$ and $\pi_{m}^{*}$, and turns out to be intuitively easy to interpret. As shown in Figure 2(a), the AAI prefers to buy more reinsurance if she is more ambiguity-averse. Moreover, she tends to increase her retention with the increase of reinsurance premium, as shown in Figure 2(b) via the safety 
loading factor $1+\theta$. Moreover, Figure 2 uncovers an important impact of time $t$ on the optimal reinsurance. For short time horizon, the AAI tends to purchase more reinsurance and transfer more risk to the reinsurer; but for long time horizon, the AAI may transfer little to the reinsurer, especially when the loading factor of the reinsurance is very high, where the AAI may refuse reinsurance altogether, as seen Figure 2(b). These points quantitatively highlight the intuitive fact that, in an unfavorable reinsurance market, one should avoid over-reliance on reinsurance, judging by the low optimal levels illustrated in these figures, but that on the other hand, model ambiguity on the insurance-surplus side should result in a greater reliance on reinsurance, which is also intuitive.

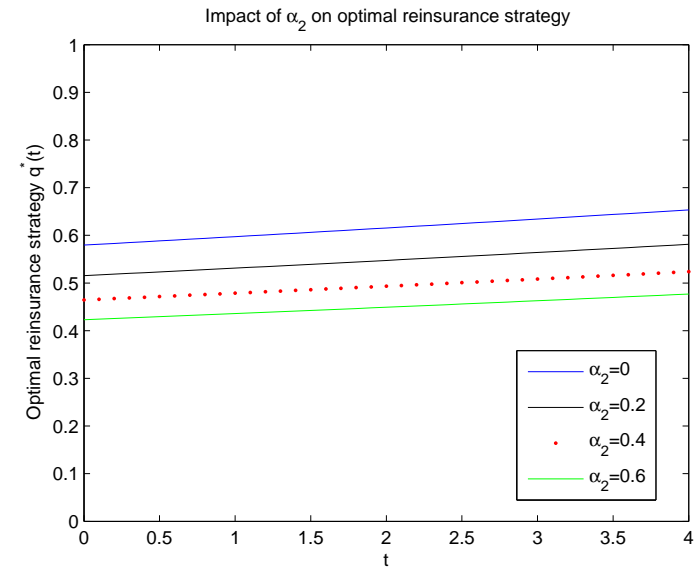

(a)

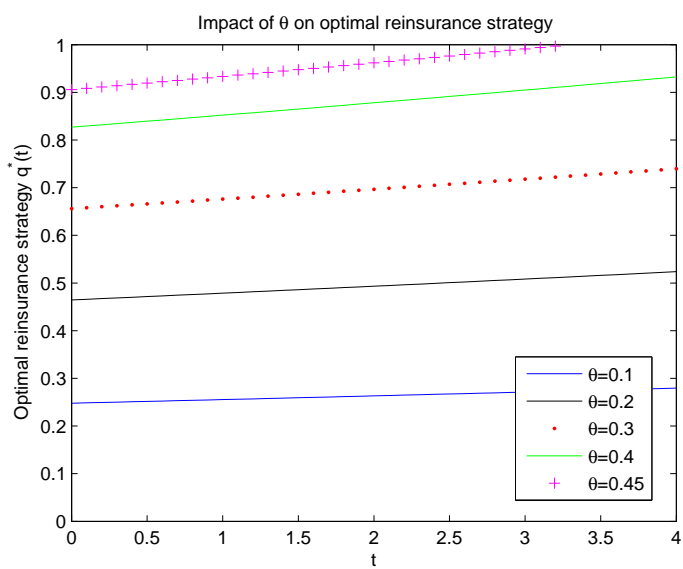

(b)

Figure 2: Impact of $\alpha_{2}$ and $\theta$ on the optimal reinsurance strategy

\subsection{Impact of parameters on utility loss function}

From the analysis of our optimal investment and reinsurance strategy, we know that the AAI will take a more conservative strategy than the ANI. If she does so, it will cause less expected utility comparing to the ANI's. Similarly, by choosing to use a jump-surplus C-L model, the AAI will get less expected utility than by using a diffusion approximation model, i.e. with no jumps. In this subsection, one objective is to quantify and analyze these decreases in utility by introducing below the utility loss functions $L_{1}$ and $L_{2}$ that the AAI is willing to take by accepting the ambiguity aversion and by setting up the model with surplus jumps. We also investigate the the utility loss from ignoring mispricing or reinsurance, via $L_{3}$ and $L_{4}$ respectively defined below.

First, we study the utility deviation from accepting model uncertainty. According to the value function $J_{0}$ without model distortion or ambiguity aversion, we define the following utility loss function:

$$
L_{1}:=1-\frac{J_{0}}{J}
$$


where $J$ is the optimal value function for the AAI . An AAI is suspicious about the reference model; recall that $\left(\alpha_{1}, \alpha_{2}\right)$ represents the ambiguity aversion parameters to the reference model. As discussed in Section 5.1, due to the existing of uncertainty in the model, the AAI prefers to take more conservation investment and reinsurance strategies when she is more ambiguity averse. Therefore, the AAI has to accept some utility loss comparing to the optimal value function $J_{0}$ without ambiguity. As shown in Figure $3(\mathrm{a}-\mathrm{b})$, the utility loss will increase with the increase of ambiguity aversion level $\left(\alpha_{1}, \alpha_{2}\right)$ and decrease with time. An interesting phenomenon can be observed that the utility loss $L_{1}(t)$ is very sensitive to $\alpha_{1}$, but not sensitive to $\alpha_{2}$. This can be explained by the fact that most of the insurer's profits come from investments and our utility loss mainly stems from the loss of profit. In other word, being wary of ambiguity towards the insurance risk is not a critical component of the surplus investment strategy.

In relation to $L_{1}$ and to whether or not it might be worth taking this utility loss, another interesting point comes from the result of Proposition 4.3 in Section 4. There we found very strong evidence against being an ANI in some circumstances. Indeed, this proposition shows that under certain parameter choices, the utility cost of following a strategy from an incorrect model can be extreme. The loss of utility by such an erroneous modeling choice while ignoring ambiguity would be $100 \%$ since the expected exponential utility is $-\infty$. As explained in the proof of that proposition, this extreme result is due to the lack of exponential moments, which is itself caused by heavy left-hand tails in the wealth process due to the presence of a quadratic term in the variable $X$. The statement of the proposition explicitly provides conditions on the parameters under which this phenomenon occurs. It is easy to see that it occurs for small enough liquidity $\ell=l_{1}+l_{2}$. Indeed, specifically, for small $\ell$, the first condition in Proposition 4.3 is satisfied, and bracketed term in the second condition in Proposition 4.3 is equivalent to $(t+T) /(T-t)$, which means that for small $\ell$, that second condition becomes approximately

$$
2(2 a b-\ell)\left(T+\ell^{-1}\right)(t+T) \geq T-t,
$$

which is easily satisfied for all small $\ell$. In conclusion here, for small liquidity, the conditions of Proposition 4.3 are easily satisfied, causing the use of incorrect models to be extremely dangerous for the ANI, giving more incentive for the insurer to choose to be an AAI, since the utility loss $L_{1}$ is much less drastic according to Figure 3.

Next, we discuss the utility loss an AAI has to accept by adopting surplus jumps in the insurance model. Define the utility loss function

$$
L_{2}:=1-\frac{\breve{J}}{J} .
$$




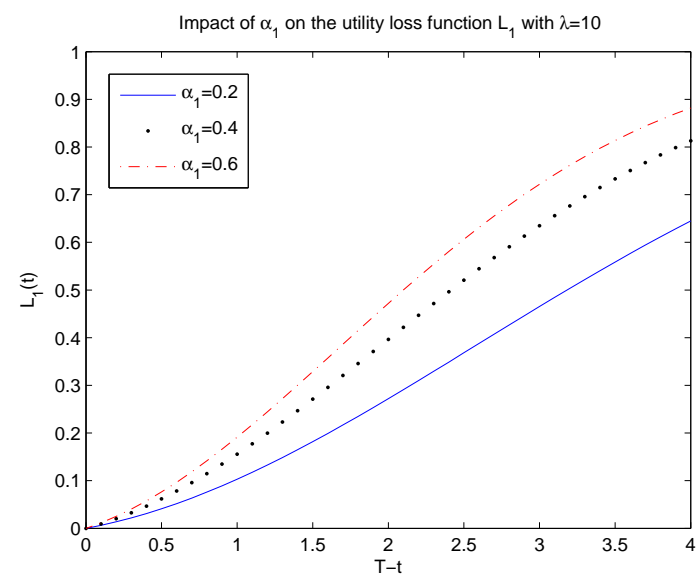

(a)

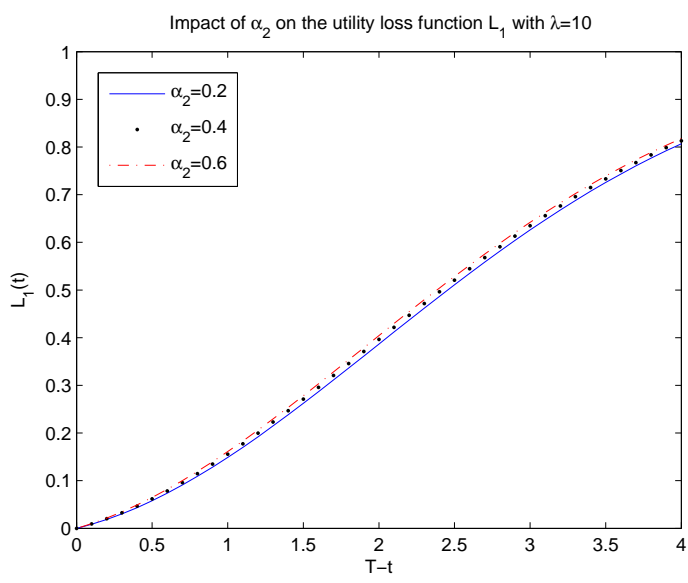

(b)

Figure 3: Impact of $\alpha_{1}$ and $\alpha_{2}$ on the loss utility $L_{1}$

The impact on $L_{2}$ of ambiguity aversion $\alpha_{2}$ and the intensity of claims $\lambda$ is shown by Figure 4, which tells us that ignoring jump term surely brings more profits, but comes with more risk. As shown in that figure, the utility loss will increase when claims become heavier (increase of claim intensity $\lambda$ ). Here the graphs show that for any horizon above a few months, ignoring the jumps in the model is a very foolish idea. Instead, the AAI will prefer to use the jump model to seek a more conservative strategy, rather than to pursue higher profit with a possibly ill-approximating diffusion model. This is consistent with the intuition that safety consciousness forces the risk exposure downward. Another phenomenon is that the utility loss is not sensitive to the ambiguity aversion $\alpha_{2}$, the reason is same as for why utility loss $L_{1}$ is not sensitive to $\alpha_{2}$. Comparison of Figure 3(b) and Figure 4(a) shows that it is much more dangerous to ignore the jump component of the model than to take a loss by incorporating ambiguity into the intensity of the jump component via the parameter $\phi$. Therefore, if the insurer is worried about not being able to compute or estimate $\lambda$, she should use ambiguity robustness, not ignore $\lambda$ in favor of a diffusion model.

Next, we focus on the utility loss from ignoring mispricing in the pair of stocks. This means that the AAI is willing to invest in risky assets, but only by investing market indices, not by trying to take advantage of the individual statistical arbitrages present in mispriced stocks. As shown in Proposition 4.4, $\hat{J}$ is the optimal value function for problem (35) where the investment in the pair of stock prices is not considered. Due to the absence of investment in these assets, $\hat{J}$ is less than $J$. Thus the utility loss function from ignoring mispricing can be defined as

$$
L_{3}:=1-\frac{J}{\hat{J}} .
$$




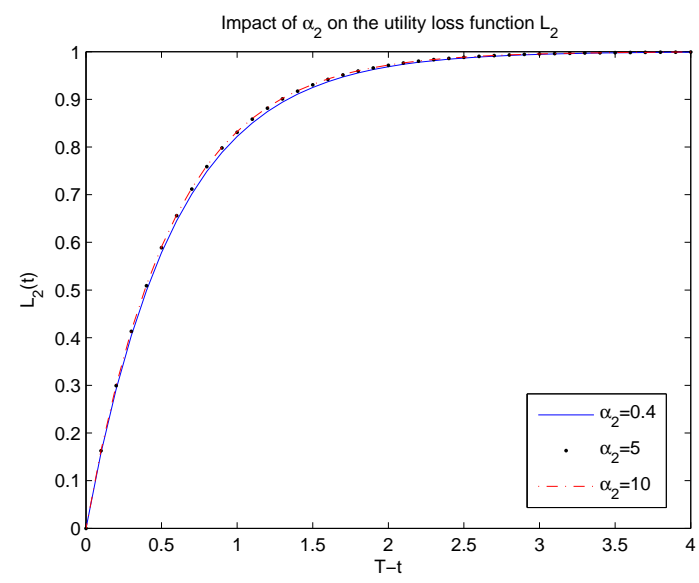

(a)

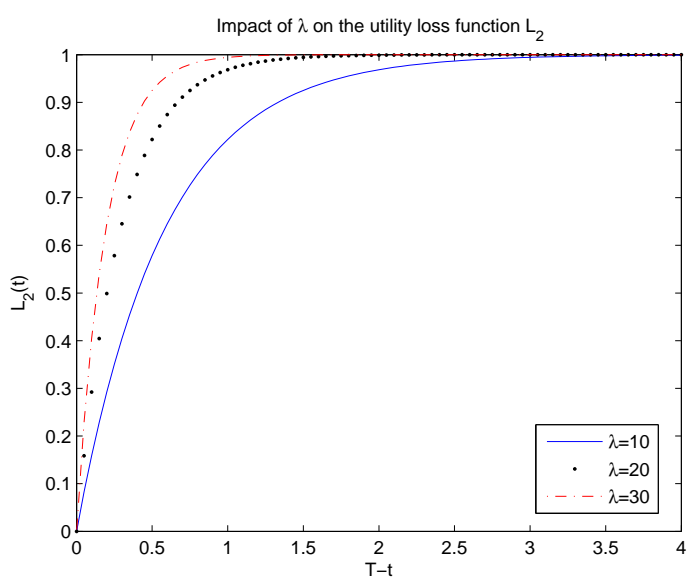

(b)

Figure 4: Impact of $\alpha_{2}$ and $\lambda$ on the utility loss $L_{2}(t)$

This immediately computes as

$$
L_{3}=1-\exp \left\{-\gamma\left[Q(t)-\hat{Q}(t)+\frac{1}{2} M(t) x^{2}\right]\right\},
$$

where $Q(t), \hat{Q}(t)$ and $M(t)$ are given by Eq (25) and Proposition 4.4. We see that the utility loss $L_{3}$ is independent of the wealth process and dependent on the mispricing level $x$. Also, it is an increasing function of $x$. Fixing $t=2$ and varying $x$ from 0 to 0.2 , Figure 5 (a-c) illustrates the change of utility loss with $x$. If we fix $x=0.2$, the utility loss will increase with the $T-t$, which can be seen in Figure 5(d). Figure 5(a) shows the impact of individual stock risk volatility $b$ on the utility loss. If there is mispricing, and $b$ is small, the insurer has to take advantage of it: for instance when $x=0.2$ and $b=0.1$, the utility loss is close to 0.9 which means it would be extremely unfortunate to ignore the mispricing investment opportunity. Even for higher values of $b$, the utility loss by ignoring mispricing is still quite substantial, exceeding $50 \%$ for all initial values of the mispricing $x$. However, variations in the common stock risk volatility $\sigma$ does not generate major changes in the utility loss, as shown in Figure 5(b) with $b=0.3$, i.e., the utility loss is not sensitive to $\sigma$. Comparing Figure $5(\mathrm{a})$ and (b), $b$ is an important factor in making a decision whether to pursue mispricing arbitrages. Similarly, $l_{1} X(t)$ and $l_{2} X(t)$ are error correction terms in the price processes of the pair of stocks, which have important impact on the utility loss, which can be illustrated by Figure 5 (c) with $t=2$ and $x$ changing from 0 to 0.2 and Figure 5 (d) with $x=0.2$ and $T-t$ changing from 0 to 4 . Comparing these two graphs, we find that the investment horizon is the key factor in utility loss: the loss is very small for a one-year horizon, but it is very large for a four-year horizon. We should note that the mispricing process $X$ is mean-reverting, which visit all the value in its typical range around 0 , no matter what $X(0)$ 
is, as long as $T-t$ is big enough, or as long as the rate of mean reversion (i.e., $\left.l_{1}+l_{2}\right)$ of $X$ is big enough. Therefore, as per Figure $5(\mathrm{c}-\mathrm{d})$, we see that taking advantage of mispricing can provide high profits for longer-term horizons. This is consistent with the result of Liu and Longstaff (2004). In plain language, short-term investment strategies, of less than a year, need not typically attempt to exploit market mispricing, but any medium or long-term investment strategy (horizon longer than 3 years in our market-calibrated scenarios) would be ill-advised to ignore mispricing, and extremely so if market mispricing reverts back to the zero mean quickly (large $l_{1}+l_{2}$ ). In fact, the change in utility loss is so dramatic, either as the time horizon goes from 1 year to 3 years, or as the mispircing mean reversion rate increases from 0.2 to 0.4 , that one can almost talk about a phase transition, going from a regime where not taking advantage of mispricing is inoccuous, to one where it is a critical investment error.

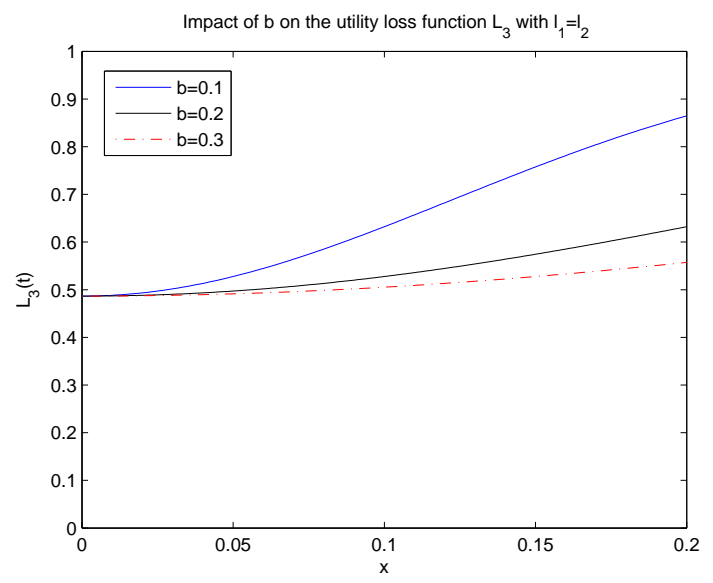

(a)

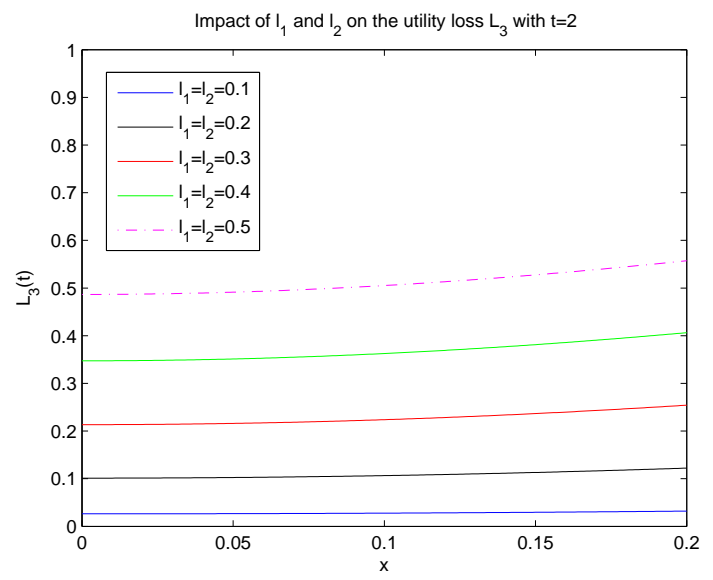

(c)

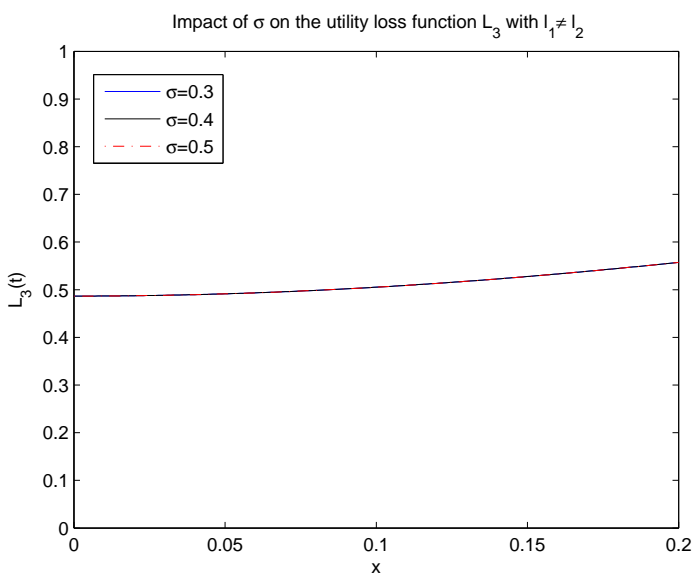

(b)

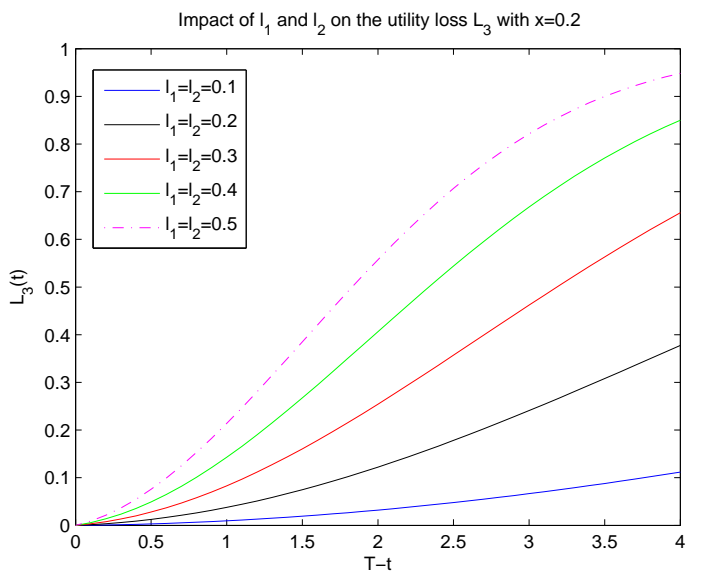

(d)

Figure 5: Impact of $b, \sigma, l_{1}$ and $l_{2}$ on the loss utility $L_{3}(t)$

Finally, we analyze utility loss from ignoring reinsurance. According to value function $\tilde{J}$ 
given in Proposition 4.5, denote utility loss function

$$
L_{4}:=1-\frac{J}{\tilde{J}}=1-\exp \{-\gamma(Q(t)-\tilde{Q}(t))\}
$$

where $Q(t), \tilde{Q}(t)$ is given by $\mathrm{Eq}(25)$ and Proposition 4.5, respectively. Figure 6 shows the impact of ambiguity aversion $\alpha_{2}$, the claim intensity $\lambda$ and safety loading of the reinsurer $\theta$ on utility loss, and shows that there is no need to buy reinsurance when investment horizon time $T-t$ is small, but for a long investment horizon, reinsurance is typically very important and cannot be ignored. Figure 6 (a) and (b) indicates that utility loss will increase with the increase of $\alpha_{2}$ and $\lambda$, which means that the AAI prefers to transfer part of risk to the reinsurer when she is more ambiguity-averse or when the claim intensity becomes larger. On the question of claim intensity, we have chosen to present results in Figure 6(b) for rather high intensities, up to $\lambda=30$, which means that the insurer is expecting about 30 claims per year. This represents relatively realistic scenarios for insurance markets with large infrequent claims, which are well adapted to being modeled with the compound Poisson process we have chosen (recall our classical Cramer-Lundberg model (1) of Section 2.1, with reinsurance). When $\lambda$ becomes significantly larger than 30, this model could be approximated by a diffusion model with no jumps, as a normal approximation regime kicks in, which changes the model class; this is why we avoid presenting models with a value of lambda beyond 30 . On the topic of the cost of reinsurance, intuition tells us that the insurer would like to decrease or give up reinsurance with the increase of reinsurance premium. As a consequence we would expect the utility loss decline with the increase of the reinsurance premium. This is indeed what we observe in Figure 6(c). In fact, recall from Figure 2(b) that the insurer begins to abandon her position in reinsurance when the loading factor $1+\theta$ exceeds $140 \%$; we see this

effect in Figure 6(c) again, where foregoing reinsurance entirely at a factor of $140 \%$ implies essentially no loss of utility, and the loss is rather moderate at $130 \%$ even for long horizon.

\section{Conclusions}

In this paper, we investigated optimal reinsurance-investment strategies for an ambiguityaverse insurer (AAI) with market mispricing investment opportunities such as those which exist between stock markets in Hong Kong and Shanghai. The surplus process of the AAI is described by the Cramer-Lundberg model and the insurer has ambiguity aversion to the jump term in that model. The financial market consists one risk-free asset, one market index and a mispriced pair of stocks which offer statistical arbitrage opportunities; diffusion models are used for the risk assets, with the mispricing ratio being modeled as a Gaussian meanreverting stochastic process whose mean-reversion rate responds to liquidity constraints. The insurer is skeptical about the insurance and financial models, in the sense that she worries about the consequences of using an incorrect model; this can also be interpreted as 


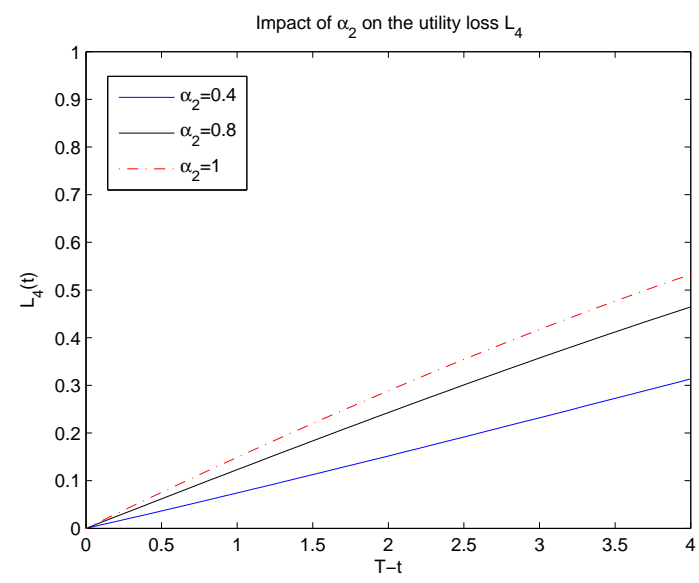

(a)

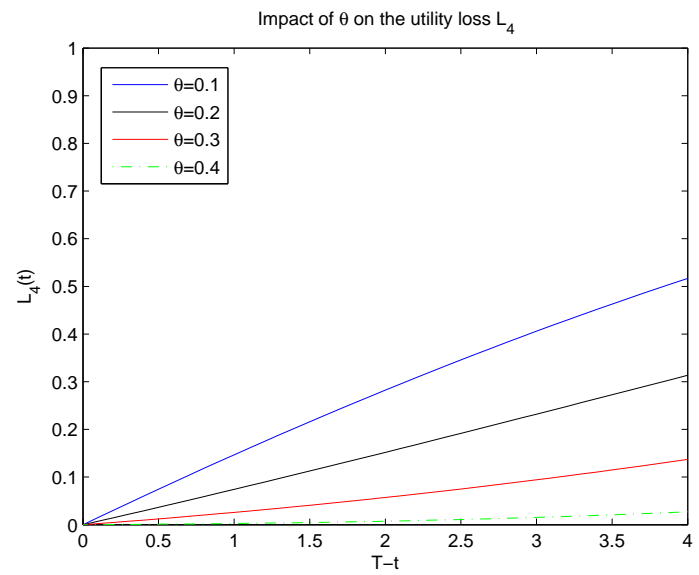

(c)

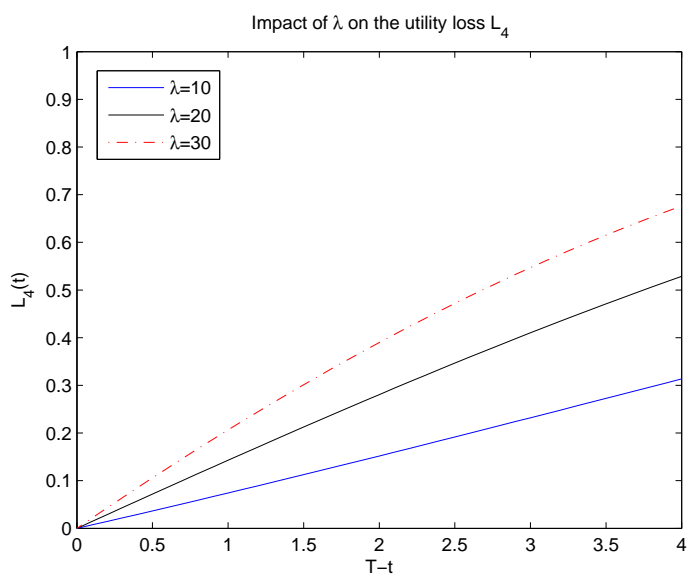

(b)

Figure 6: Impact of $\alpha_{2}, \lambda$ and $\theta$ on the loss utility $L_{4}(t)$

an aversion to her lack of confidence in being able to estimate these models' jump intensity and drift parameters accurately. She chooses to hedge partially against this modeling risk by using a "homothetic robust" utility, which incorporates the ambiguity aversion into the model in a fully quantitative way. By employing the dynamic programming approach, we derive explicitly the optimal robust reinsurance-investment strategy and its corresponding optimal value function.

We use these explicity formulae in a detailed numerical illustration to analyze the formulae's behavior and make practical recommendations. By studying our portfolio allocation sensitivity to various parameters, among other things, we uncover and analyze complex behavior resulting from asymmetry between the liquidities/reversion rates of the mispriced stock, while when these stocks have identical reversion rates, interpretating investment positions as depending on ambiguity-aversion levels is more straightforward. We also define and analyze various utility losses which explain the importance of ambiguity aversion, surplus- 
jump, mispricing, and reinsurance in our model. It is a mathematical fact that ignoring ambiguity aversion and surplus jumps bring higher profits if the base model is correct, i.e. that accepting ambiguity robustness comes at a loss of utility, but taking the modeling risk of ignoring ambiguity can be very unwise in a number of different circumstances, particularly for relatively illiquid mispriced markets. Comparing Figure 3 and Figure 4, we find that ignoring the jump term is more dangerous than ignoring ambiguity aversion. We also find that the ambiguity aversion tuning parameter $\alpha_{1}$ is more important in the view of profits, but allowing for a jump term in the surplus process is a key factor in the view of avoiding risk. Figure 5 and Figure 6 tell us that not taking advantage of mispricing and reinsurance opportunities can cause significant losses in profits. In fact, the impacts of taking advantage of these opportunities are greater for longer investment horizons. Specifically, they should never be ignored for investment horizons of 3 or more years, while for shorter horizons of a year or less, reinsurance can typically be ignored with little loss of utility; we even show that, under our market-calibrated parameters, the AAI can safey forego reinsurance completely if the reinsurer's loading factor exceeds $140 \%$.

\section{References}

[1] Anderson, E., Hansen, L., Sargent, T., Robustness detection and the price of risk.Working paper, University of Chicago. Available at: https://files.nyu.edu/ts43/public/research/.svn/text-base/ahs3.pdf.svn-base.

[2] Branger, N., Larsen, L.S., 2013. Robust portfolio choice with uncertainty about jump and diffusion risk. J. Bank. Finance 37 (12), 5036-5047.

[3] Bäuerle, N., Benchmark and mean“Cvariance problems for insurers. Math. Methods Oper. Res, 2005, 62 (1), 159-165.

[4] Chen S M and Li Z F, Optimal investment-reinsurance strategy for an insurance company with VaR contraint. Insurance: Mathematics and Economics, 2010, 47, 144-153.

[5] Grandell, J., Aspects of risk theory. Springer-Verlag, New York, 1991.

[6] Gerber, H., An extension of the renewal equation and its application in the collective theoty of risk. Skandinavina Actuarial Journal, 1970, 205-210.

[7] Gu A L, Yi B and Ye D Z, Optimal investment and reinsurance for insurers with uncertain time-horizon. Mathematical Problems in Engineering, 2014, http://dx.doi.org/10.1155/2014/271930.

[8] Jurek, J.W., Yang, H.: Dynamic portfolio selection in arbitrage.Working paper, 2006, Harvard University. 
[9] Korn, R., Menkens, O., Steffensen, M., Worst-case-optimal dynamic reinsurance for large claims. Eur. Actuar. J. 2012, 2 (1), 21-48.

[10] Liu, J., Pan, J., Wang, T., An equilibrium model of rare-event premia and its implication for option smirks, Rev. Financ. Stud. 2005, 18, 131-164.

[11] Liu, H. N., Robust consumption and portfolio choice for time varying investment opportunities. Annals of Finance, 2010, 6, 435-454.

[12] Liu, J., Longstaff, F.A.: Losing money on arbitrage: optimal dynamic portfolio choice in markets with arbitrage opportunities. Review of Financial studies, 2004, 17, 611-641.

[13] Liu, J., Timmermann, A., Optimal convergence trade strategies. Review of Financial Studies, 2013, 26, 1048-1086.

[14] Maenhout, P. J., Robust portfolio rules and asset pricing. The Review of Financial Studies, 2004, 17, 951-983.

[15] Maenhout, P. J., Robust portfolio rules and detection-error probabilities for a meanreverting risk premium. Journal of Economic Theory, 2006, 128, 136-163.

[16] Mataramvura, S., Øksendal, B., Risk minimizing portfolios and HJBI equations for stochastic differential games. Stochastics: An International Journal of Probability and Stochastics Processes, 2008, 80, 317-337.

[17] Munk, C., Rubtsov, A., Portfolio management with stochastic interest rates and inflation ambiguity. Ann. Finance, 2014, 10 (3), 419-455.

[18] Schmidli, H., Optimal proportional reinsurance policies in a dynamic setting. Scandinavian Actuarial Journal, 2001, 1(1), 55-68.

[19] Shen Y and Zeng Y, Optimal investment-reinsurance with delay for mean-variance insurers: A maximum principle approach. Mathematical Finance, 2014, 57, 1-12.

[20] Uppal, R and Wang, T., Model misspecification and underdiversification. The Journal of Finance, 2003, 58, 2465-2486.

[21] Xu, L., Wang, R.M., Yao, D.J., On maximizing the expected terminal utility by investment and reinsurance. Journal of Industrial and Management Optimization, 2008, 4, 801-815.

[22] Yi B, Viens F, Li Z F and Zeng Y, Robust optimal strategies for an insurer with reinsurance and investment under benchmark and mean-variance criteria, Scandinavian Actuarial Journal, 2015, 8, 725-751. 
[23] Yi B, Li Z F, Viens F and Zeng Y, Robust optimal control for an insurer with reinsurance and investment under Heston's stochastic volatility model, Insurance: Mathematics and Economics, 2013, 53,601-614.

[24] Zeng, Y., Li, D.P., Gu, A. L., Robust equilibrium reinsurance-investment strategy for a mean-variance insurer in a model with jumps. Insurance: Mathematics and Economics, 2016, 66, 138-152.

[25] Zhang, X., Siu, T. K., Optimal investment and reinsurance of an insurer with model uncertainty. Insurance: Mathematics and Economics, 2009, 45, 81-88.

[26] Zhou, X., On a classical risk model with a constant dividend barrier, North Amercian Actuarial Journal, 2005, 95-98. 